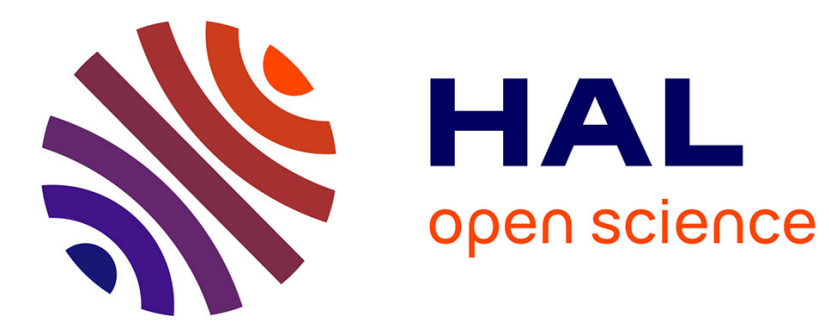

\title{
Speed limit and ramp meter control for traffic flow networks
}

\author{
Paola Goatin, Simone Göttlich, Oliver Kolb
}

\section{To cite this version:}

Paola Goatin, Simone Göttlich, Oliver Kolb. Speed limit and ramp meter control for traffic flow networks. Engineering Optimization, 2015, 10.1080/0305215X.2015.1097099 . hal-01234592

\section{HAL Id: hal-01234592 \\ https://hal.science/hal-01234592}

Submitted on 27 Nov 2015

HAL is a multi-disciplinary open access archive for the deposit and dissemination of scientific research documents, whether they are published or not. The documents may come from teaching and research institutions in France or abroad, or from public or private research centers.
L'archive ouverte pluridisciplinaire HAL, est destinée au dépôt et à la diffusion de documents scientifiques de niveau recherche, publiés ou non, émanant des établissements d'enseignement et de recherche français ou étrangers, des laboratoires publics ou privés. 


\title{
Speed limit and ramp meter control for traffic flow networks
}

\author{
Paola Goatin*, Simone Göttlich,* Oliver Kolb**
}

June 16, 2015

\begin{abstract}
The control of traffic flow can be related to different applications. In this work, we introduce how to manage variable speed limits combined with coordinated ramp metering within the framework of the LWR network model. Following a "first discretize then optimize" approach, we derive the first order optimality system and explain the switch of speeds at certain fixed points in time and the boundary control for the ramp metering as well. Sequential quadratic programming methods are used to solve the control problem numerically. For application purposes, we present experimental setups where variable speed limits are used as a traffic guidance system to avoid traffic jams on highway interchanges and on-ramps.
\end{abstract}

AMS Classification. 90B20, 49K20

Keywords. Traffic flow network, control of discretized PDEs, adjoint based optimization

\section{Introduction}

Traffic flow networks based on scalar conservation laws have been investigated from various perspectives during the last years, see for instance $[5,18]$ for theoretical results or $[2,31]$ for numerical considerations and applications. In the case of nonlinear traffic flow networks where the dynamics obey the Lighthill-WhithamRichards equations $[23,27]$, the crucial point is the modeling of intersections. Assuming conservation of mass, further conditions like flux maximization at nodes need to be prescribed to obtain unique admissible solutions. This approach results in appropriate Riemann solutions that can be directly specified, see [8]. We will use this mathematical formulation and framework to describe the dynamics for the traffic system.

For many applications, not only the numerical simulation of the nonlinear dynamics is of interest but also optimization or control issues. To set up the optimization problem, a cost functional and control variables must be identified, while the constraints are given by the above mentioned traffic flow network equations. There exists a broad range of practical aspects including the

\footnotetext{
*INRIA Sophia Antipolis - Méditerranée, 06902 Sophia-Antipolis, France (paola.goatin@inria.fr).

** University of Mannheim, Department of Mathematics, 68131 Mannheim, Germany (\{goettlich, kolb\}@uni-mannheim.de).
} 
optimal routing of traffic [13], traffic light control [12] or coordinated ramp metering [26] for the LWR network model. Since these optimal control problems are constrained by nonlinear conservation laws, relaxed models with simplified dynamics have been investigated instead, cf. [17].

Reduced or time-discrete models usually rely on linear approximations leading to control methodologies such as linear optimization [24], feedback control $[4,25]$ or Lyapunov stability [1], respectively. However, nonlinear control techniques as for instance adjoint calculus [26] or model predictive control [14] represent a suitable and natural choice to treat traffic network control problems.

We concentrate on the variable speed limit (VSL) problem coupled to ramp metering. From a mathematical point of view, the control variable, i.e. the maximal velocity for the VSL problem, varies and must be evaluated at discrete points in time. This leads to conservation laws with time-dependent discontinuous coefficients [6]. On the other hand, the on-ramp metering problem corresponds to a discussion of boundary conditions at junctions [9,10]. Setting up the corresponding control problems is then a combination of two control issues directly influencing each other in the sense that time-dependent speed limits reduce congestions and hence the travel times. This kind of problem for macroscopic traffic flow models have been for example considered in $[4,7,14,24]$ and references therein. In contrast to our approach, the latter either follow a model predictive control [14] where the optimization is obtained using sequential quadratic programming (SQP), closed loop/feedback control $[4,7]$ or linear programming techniques [24]. Mostly, these approaches are restricted to the presentation of (numerical) results for one road/one on-ramp only while complex road network topologies are not considered.

To the best of our knowledge, the variable speed limit control problem combined with ramp metering has never been solved for the full LWR model on complex network topologies in a rigorous mathematical manner. In this article, we will close this gap from a modeling and computational point of view. We will stick to continuous optimization techniques where usually the first order optimality system is derived and solved by a descent type method [19,28]. We apply the so-called discretize-then-optimize approach, meaning that a suitable numerical discretization is chosen such that the original problem leads to a finite-dimensional optimality system [13]. In this way, we automatically include information about the current traffic situation into the control framework (open loop control) and look for the best traffic management. This procedure was originally introduced for aerodynamic shape design [11].

We proceed as follows: We briefly recall the LWR network model in Section 2. Then, in Section 3, we explain how we model variable speed limits and ramp metering. We address the optimal control problem and its solution procedure by discretizing the governing equations in space and time. The numerical results are collected in Section 4. In particular, we point out benefits and drawbacks of our approach. We also observe that variable speed limits and ramp metering help to navigate the traffic flow, so that congestion cannot always be prevented but reduced. 


\section{Traffic Flow Network Modeling}

Following the ideas in $[5,8]$, we typically model a traffic flow network as a directed graph $G=(V, E)$, where the edges $E$ correspond to roads and the vertices $V$ to junctions or intersections. Each edge $e \in E$ is associated with an interval $\left[0, L_{e}\right]$ and $\rho_{e}(x, t)$ denotes the density of cars on road $e$.

Given some initial state $\rho_{e}(x, 0)$ on all roads, the dynamics are described by

$$
\partial_{t} \rho_{e}(x, t)+\partial_{x} f_{e}\left(\rho_{e}(x, t), t\right)=0 \quad \forall e \in E, x \in\left(0, L_{e}\right), t \in[0, T]
$$

with Lighthill-Whitham-Richards flux [23,27]

$$
f_{e}(\rho, t)=\rho v_{e}^{\max }(t)\left(1-\frac{\rho}{\rho_{e}^{\max }}\right),
$$

where $v_{e}^{\max }(t)$ is the (piecewise constant) maximal speed limit and $\rho_{e}^{\max }$ is the maximal car density corresponding to the jammed situation. See for instance Figure 1 for an illustration of the dependencies of the velocity and the flow rate with respect to the density in case of different speed limits. We also denote by $f_{e}^{\max }(t)$ the maximal flux:

$$
f_{e}^{\max }(t)=f_{e}\left(\rho_{e}^{c}, t\right) \text { with } \rho_{e}^{c}=\rho_{e}^{\max } / 2 .
$$
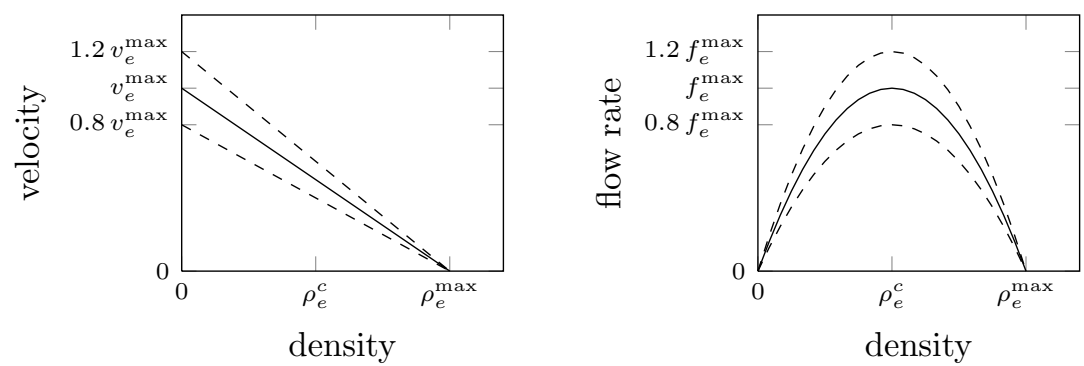

Figure 1: Velocity and flow rate for different speed limits.

We also see in Figure 1 that different maximal velocities $v_{e}^{\max }(t)$ lead to conservation laws on networks with time-dependent discontinuous coefficients. Note that general existence results for these type of conservation laws on networks are not available at the moment. The network consideration requires the extension of the well-posedness result given in [6] for the standard Cauchy problem on the real line. Nevertheless, in the specific application considered in this work, the time-dependence is piecewise constant, and therefore classical existence results on networks can be applied [9].

\section{Coupling Conditions at Junctions}

The description of the whole network dynamics requires the definition of the corresponding boundary and coupling conditions at junctions $v \in V$ to basically ensure the conservation of mass. Therefore, we introduce the demand and supply functions that are given respectively by

$$
D_{e}(\rho, t)=\left\{\begin{array}{ll}
f_{e}(\rho, t) & \text { if } \rho \leq \rho_{e}^{c}, \\
f_{e}^{\max }(t) & \text { if } \rho \geq \rho_{e}^{c},
\end{array} \quad \text { and } \quad S_{e}(\rho, t)= \begin{cases}f_{e}^{\max }(t) & \text { if } \rho \leq \rho_{e}^{c}, \\
f_{e}(\rho, t) & \text { if } \rho \geq \rho_{e}^{c}\end{cases}\right.
$$


For simplicity and in view of the applications considered in this work, we will only consider the cases of one-to-one junctions, the merging of two roads into one, and the dispersing of one road into two, see Fig. 2.

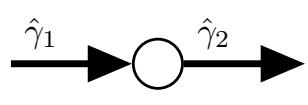

(a) One-to-one

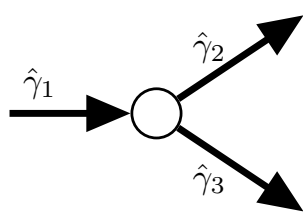

(b) One-to-two

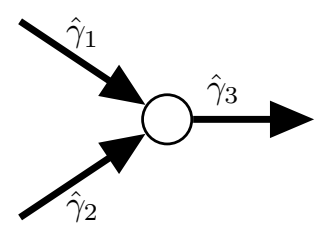

(c) Two-to-one

Figure 2: Different types of junctions.

In the case of a junction between only an incoming road $e=1$ and an outgoing one $e=2$, the appropriate fluxes at the junction are simply obtained through the minimization between demand and supply:

$$
\hat{\gamma}_{1}=\hat{\gamma}_{2}=\min \left\{D_{1}\left(\rho_{1}, t\right), S_{2}\left(\rho_{2}, t\right)\right\}
$$

Note that we explicitly indicate the time dependency of the demand and supply functions here, but we omit the dependencies for all $\gamma \mathrm{s}$ for a better reading.

Let us now consider more involved cases, e.g. the case of a dispersing junction with one incoming road $e=1$ and two outgoing roads $e=2, e=3$. Here, the distribution of cars must be externally prescribed by so-called distribution rates $\alpha_{2,1} \geq 0$ and $\alpha_{3,1} \geq 0$ (and $\alpha_{2,1}+\alpha_{3,1}=1$ ).

Contrarily to what is proposed in [8], we make the choice of applying NONFIFO rules, thus allowing for some flow through the junction even if one of the outgoing roads is blocked. This is more reasonable for highway networks than a classical FIFO model. The fluxes at the junction are computed as (see also $[16,22])$

$$
\begin{aligned}
& \hat{\gamma}_{2}=\min \left\{\alpha_{2,1} D_{1}\left(\rho_{1}, t\right), S_{2}\left(\rho_{2}, t\right)\right\}, \\
& \hat{\gamma}_{3}=\min \left\{\alpha_{3,1} D_{1}\left(\rho_{1}, t\right), S_{3}\left(\rho_{3}, t\right)\right\}, \\
& \hat{\gamma}_{1}=\hat{\gamma}_{2}+\hat{\gamma}_{3} .
\end{aligned}
$$

Finally, we focus on the case of a merging junction of ingoing two roads $e=1, e=2$ and outgoing one $e=3$. In this case, a priority parameter $P \in(0,1)$ is introduced so that $\hat{\gamma}_{1}=P \hat{\gamma}_{3}$ and $\hat{\gamma}_{2}=(1-P) \hat{\gamma}_{3}$. This is necessary to define a unique solution in the supply constrained cases, see [8, Section 5.2.2] for more details. The corresponding fluxes are then

$$
\begin{aligned}
& \hat{\gamma}_{1}=\min \left\{D_{1}\left(\rho_{1}, t\right), \max \left\{P S_{3}\left(\rho_{3}, t\right), S_{3}\left(\rho_{3}, t\right)-D_{2}\left(\rho_{2}, t\right)\right\}\right\}, \\
& \hat{\gamma}_{2}=\min \left\{D_{2}\left(\rho_{2}, t\right), \max \left\{(1-P) S_{3}\left(\rho_{3}, t\right), S_{3}\left(\rho_{3}, t\right)-D_{1}\left(\rho_{1}, t\right)\right\}\right\}, \\
& \hat{\gamma}_{3}=\hat{\gamma}_{1}+\hat{\gamma}_{2} .
\end{aligned}
$$

The model equations (1)-(4) can be further coupled to inflow conditions at roads entering or leaving the network. As we will see later on, this modeling framework can be extended to describe the ramp metering. 


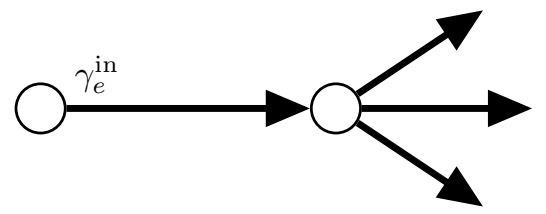

Figure 3: Road with an inflow condition.

\section{Inflow and Outflow Conditions}

Let us consider an arc $e$ connected to the network only at its right node as shown in Figure 3. Given a desired inflow rate $f_{e}^{\text {in }}(t)$, the actual inflow to the $\operatorname{road} e$ is given by

$$
\gamma_{e}^{\text {in }}=\min \left\{f_{e}^{\text {in }}(t), S_{e}\left(\rho_{e}, t\right)\right\} .
$$

We assume there is a queue at the left node of $\operatorname{arc} e$ with length $l_{e}(t)$. Then, the inflow to the road is given by

$$
\gamma_{e}^{\text {in }}=\min \left\{D_{e}\left(l_{e}, t\right), S_{e}\left(\rho_{e}, t\right)\right\}
$$

with the demand function dependent on the current length $l_{e}(t)$ and the time $t$,

$$
D_{e}\left(l_{e}, t\right)= \begin{cases}\tilde{f}_{e}^{\max } & \text { if } l_{e}>0 \\ \min \left\{f_{e}^{\text {in }}(t), \tilde{f}_{e}^{\max }\right\} & \text { if } l_{e}=0 .\end{cases}
$$

Here, $\tilde{f}_{e}^{\max }$ denotes the maximum flux from the queue onto the road. As usual, the evolution of the queue length $l_{e}(t)$ obeys the rate equation

$$
\frac{d l_{e}(t)}{d t}=f_{e}^{\text {in }}(t)-\gamma_{e}^{\text {in }}
$$

for an initial state $l_{e}(0)$.

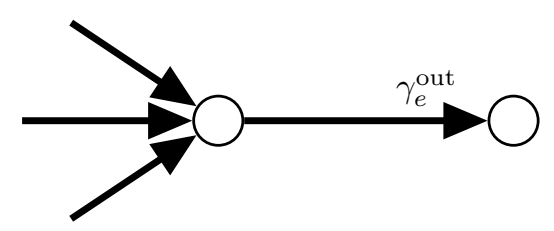

Figure 4: Road with an outflow condition.

Conversely, on arcs connected only on their left to the network as shown in Figure 4, we prescribe absorbing boundary conditions up to a given maximum flow rate $f_{e}^{\text {out }}(t)$ in the form

$$
\gamma_{e}^{\text {out }}=\min \left\{f_{e}^{\text {out }}(t), D_{e}\left(\rho_{e}, t\right)\right\},
$$

which ensures that the flow reaching the end of such a road is able to leave the network without being stopped if it is below the given maximum flow rate.

In the next section, we describe the optimization problem we intend to solve. We also comment on the discretization and the numerical implementation. 


\section{Optimal Control Problem}

For optimization purposes, we are interested in controlling the traffic flow through a network by adjusting maximal speed limits and on-ramp fluxes as well.

The overall goal of optimization is to choose the controls in such a way that the total travel time is minimized [31] and/or the outflow of the system is maximized, i.e.

$$
\min J\left(\vec{l}, \vec{\rho}, \vec{\gamma}^{\text {out }}\right):=\sum_{e} \beta_{e} \int_{0}^{T}\left(l_{e}(t)+\int_{0}^{L_{e}} \rho_{e}(x, t) d x\right) d t-\sum_{e} \varepsilon_{e} \int_{0}^{T} \gamma_{e}^{\text {out }}(t) d t
$$

with vectors $\vec{l}=\left(l_{e}\right)_{e}, \vec{\rho}=\left(\rho_{e}\right)_{e}$ and $\vec{\gamma}^{\text {out }}=\left(\gamma_{e}^{\text {out }}\right)_{e}$, or to minimize a congestion measure similar to [26], i.e.

$\min J(\vec{l}, \vec{\rho}):=\sum_{e} \beta_{e} \int_{0}^{T}\left(l_{e}(t)+\max \left\{0, \int_{0}^{L_{e}}\left(\rho_{e}(x, t)-\frac{f_{e}\left(\rho_{e}(x, t), t\right)}{v_{e, \mathrm{ref}}}\right) d x\right\}\right) d t$.

Here, $\beta_{e}$ and $\varepsilon_{e}$ denote non-negative weights and $v_{e, \text { ref }}$ a reference velocity. Note that we later consider additional terms in the objective function such that $J$ will further explicitly depend on the control variables introduced in the following. The constraints of the optimization problem are mainly determined by the traffic flow network model introduced in Section 2. Further, we consider upper bounds for queue lengths below. These inequality constraints will be explicitly passed to the applied optimization procedure and not integrated by additional penalty terms in the objective function. However, we need to explain how the two controls are integrated into the model equations. That means, we have to modify the flux in (1) for the speed limit and (8) for the ramp metering control. Since the optimization problem under consideration is constrained by differential equations, we first use suitable numerical methods to discretize the equations and then propose a nonlinear optimization technique. This results in a so-called discretize-then-optimize approach.

\section{Variable Speed Limits}

As already mentioned and shown in Figure 1, the maximal velocities $v_{e}^{\max }(t)$ on each road $e \in E$ may change in time. Therefore, we assume that $v_{e}^{\max }(t)$ is a time-dependent control variable restricted by box constraints,

$$
v_{e}^{\text {low }} \leq v_{e}^{\max }(t) \leq v_{e}^{\text {high }} \quad \forall t \in[0, T]
$$

To get a finite dimensional optimization problem and also for practical reasons, we introduce control points $\nu^{k} \in[0, T](k \in\{0, \ldots, N u\})$ and corresponding control variables $z_{e}^{k}$ for each road. We take $v_{e}^{\max }(t)$ piecewise constant on the control grid:

$$
v_{e}^{\max }(t)=z_{e}^{k+1} \quad \forall t \in\left(\nu^{k}, \nu^{k+1}\right] .
$$

The choice of the control grid will be investigated in Section 4.1. Note that, in order to avoid high fluctuations in the control function, a penalty term can be added to (10). This issue is also addressed in Section 4.1. 


\section{Ramp Metering}

The control of on-ramps can be recast in the framework of merging junctions, i.e. we consider a two-to-one merge as in Figure 2(c). We aim at controlling the main lane access from the on-ramp, where we assume that the index $e=2$ corresponds to the on-ramp, now described by a queue (7). The time-dependent control variable $w_{e}=w_{e}(t)$ is used to control the inflow from the on-ramp into the main lane by rewriting equations (4). Mathematically, we define the controlled demand function

$$
D_{e}^{c}\left(l_{e}, t\right)=w_{e}(t) D_{e}\left(l_{e}, t\right)
$$

for $D_{e}\left(l_{e}, t\right)$ given by (7). Then we plug (13) in (4) and end up with

$$
\begin{aligned}
& \hat{\gamma}_{1}=\min \left\{D_{1}\left(\rho_{1}, t\right), \max \left\{P S_{3}\left(\rho_{3}, t\right), S_{3}\left(\rho_{3}, t\right)-D_{2}^{c}\left(l_{2}, t\right)\right\}\right\}, \\
& \hat{\gamma}_{2}=\min \left\{D_{2}^{c}\left(l_{2}, t\right), \max \left\{(1-P) S_{3}\left(\rho_{3}, t\right), S_{3}\left(\rho_{3}, t\right)-D_{1}\left(\rho_{1}, t\right)\right\}\right\}, \\
& \hat{\gamma}_{3}=\hat{\gamma}_{1}+\hat{\gamma}_{2} .
\end{aligned}
$$

Correspondingly, the evolution of the on-ramp buffer changes to

$$
\frac{d l_{2}(t)}{d t}=f_{2}^{\text {in }}(t)-\hat{\gamma}_{2}
$$

where $f_{2}^{\text {in }}(t)$ is the external boundary inflow at the on-ramp.

Similar to the speed control (12) we also assume a piecewise constant control function for the ramp metering:

$$
w_{e}(t)=w_{e}^{k+1} \quad \forall t \in\left(\nu^{k}, \nu^{k+1}\right] .
$$

A reasonable extension is to put queue length limits on (15). This case is presented in Sections 4.2 and 4.3.

Summarizing, the speed limit and ramp metering control problem for traffic flow networks reads as follows:

$$
\begin{aligned}
& \min _{\vec{z}, \vec{w}} J\left(\vec{l}, \vec{\rho}, \vec{\gamma}^{\text {out }}\right) \\
& \text { s.t. } \quad(1)-(9),(12),(13)-(16)
\end{aligned}
$$

with $\vec{z}=\left(z_{e}\right)_{e}$ and $\vec{w}=\left(w_{e}\right)_{e}$. This is a nonlinear optimization problem which can be solved within an adjoint-based optimization framework. To this end, we fully discretize (17) in a straightforward way. Then, the first order optimality system and the associated gradient information is obtained by evaluations of forward and backward (or adjoint) equations. Details of this procedure can be found in Section 3.2.

\subsection{Discretization}

For the numerical solution of the described model, we consider a finite number of time points $t^{n}=n \Delta t$ with $\Delta t=\frac{T}{N t}$. Note that the $t^{n}$ may differ from the control points $\nu^{k}$ above. Further, we divide each road $e$ into $N x_{e}$ cells of equal size $\Delta x_{e}=\frac{L_{e}}{N x_{e}}$. 
Hence, the objective functions (10) and (11) are discretized by quadrature formulas as follows:

$$
\min \sum_{e} \beta_{e} \sum_{n=1}^{N t}\left(l_{e}^{n}+\sum_{j=1}^{N x_{e}} \rho_{e, j-0.5}^{n} \Delta x_{e}\right) \Delta t-\sum_{e} \varepsilon_{e} \sum_{n=1}^{N t} f\left(\rho_{e, N x_{e}}^{n}, t^{n}\right) \Delta t,
$$

and

$$
\min \sum_{e} \beta_{e} \sum_{n=1}^{N t}\left(l_{e}^{n}+\max \left\{0, \sum_{j=1}^{N x_{e}}\left(\rho_{e, j-0.5}^{n}-\frac{f_{e}\left(\rho_{e, j-0.5}^{n}, t^{n}\right)}{v_{e, \mathrm{ref}}}\right) \Delta x_{e}\right\}\right) \Delta t,
$$

where $v_{e, \text { ref }}=\frac{1}{2} v_{e}^{\text {high }}$. The constraints are discretized by following the structure of Section 2. For the discretization of (1), we use a staggered Lax-Friedrichs scheme and a first order Godunov scheme. Leaving out the index $e$ for the road for a better reading, the staggered Lax-Friedrichs scheme reads

$$
\begin{aligned}
\rho_{0.5}^{n+1}= & \frac{1}{4}\left(3 \rho_{0.5}^{n}+\rho_{1.5}^{n}\right)-\frac{\lambda}{2}\left[f\left(\rho_{1.5}^{n}, t^{n}\right)+f\left(\rho_{0.5}^{n}, t^{n}\right)-2 f\left(\rho_{0}^{n}, t^{n}\right)\right], \\
\rho_{j-0.5}^{n+1}= & \frac{1}{4}\left(\rho_{j-1.5}^{n}+2 \rho_{j-0.5}^{n}+\rho_{j+0.5}^{n}\right)-\frac{\lambda}{2}\left[f\left(\rho_{j+0.5}^{n}, t^{n}\right)-f\left(\rho_{j-1.5}^{n}, t^{n}\right)\right], \\
\rho_{N x-0.5}^{n+1}= & \frac{1}{4}\left(\rho_{N x-1.5}^{n}+3 \rho_{N x-0.5}^{n}\right) \\
& \quad-\frac{\lambda}{2}\left[2 f\left(\rho_{N x}^{n}, t^{n}\right)-f\left(\rho_{N x-0.5}^{n}, t^{n}\right)-f\left(\rho_{N x-1.5}^{n}, t^{n}\right)\right],
\end{aligned}
$$

where $\lambda=\Delta t / \Delta x$ and

$\rho_{e, j-0.5}^{n} \approx \frac{1}{\Delta x_{e}} \int_{(j-1) \Delta x_{e}}^{j \Delta x_{e}} \rho_{e}\left(x, t^{n}\right) d x \quad$ for $\quad j \in\left\{1, \ldots, N x_{e}\right\}, n \in\{0, \ldots, N t\}$.

The first order Godunov scheme is given by

$$
\begin{aligned}
\rho_{0.5}^{n+1} & =\rho_{0.5}^{n}-\lambda\left[\min \left\{D\left(\rho_{0.5}^{n}, t^{n}\right), S\left(\rho_{1.5}^{n}, t^{n}\right)\right\}-f\left(\rho_{0}^{n}, t^{n}\right)\right], \\
\rho_{j-0.5}^{n+1} & =\rho_{j-0.5}^{n}-\lambda\left[\min \left\{D\left(\rho_{j-0.5}^{n}, t^{n}\right), S\left(\rho_{j+0.5}^{n}, t^{n}\right)\right\}\right. \\
& \left.\quad-\min \left\{D\left(\rho_{j-1.5}^{n}, t^{n}\right), S\left(\rho_{j-0.5}^{n}, t^{n}\right)\right\}\right], \quad \\
\rho_{N x-0.5}^{n+1} & =\rho_{N x-0.5}^{n}-\lambda\left[f\left(\rho_{N x}^{n}, t^{n}\right)-\min \left\{D\left(\rho_{N x-1.5}^{n}, t^{n}\right), S\left(\rho_{N x-0.5}^{n}, t^{n}\right)\right\}\right] .
\end{aligned}
$$

The inflow $f\left(\rho_{0}^{n}, t^{n}\right)$ and the outflow $f\left(\rho_{N x}^{n}, t^{n}\right)$ directly result from the applied coupling and boundary conditions. Concerning (10), $f_{e}^{\text {out }}\left(t^{n}\right)$ corresponds to $f\left(\rho_{N x}^{n}, t^{n}\right)$.

\section{Coupling conditions at junctions}

At a simple junction connecting two roads $e=1$ (incoming) and $e=2$ (outgoing), we use

$$
f\left(\rho_{1, N x_{1}}^{n}, t^{n}\right)=f\left(\rho_{2,0}^{n}, t^{n}\right)=\min \left\{D_{1}\left(\rho_{1, N x_{1}-0.5}^{n}, t^{n}\right), S_{2}\left(\rho_{2,0.5}^{n}, t^{n}\right)\right\},
$$

according to (2). 
For junctions with one incoming road $e=1$ and two outgoing roads $e=2$ and $e=3$, and non-negative distribution rates $\alpha_{2,1}$ and $\alpha_{3,1}\left(\right.$ with $\alpha_{2,1}+\alpha_{3,1}=1$ ), we apply (referring to (3))

$$
\begin{aligned}
f\left(\rho_{2,0}^{n}, t^{n}\right) & =\min \left\{\alpha_{2,1} c_{1}, c_{2}\right\}, \\
f\left(\rho_{3,0}^{n}, t^{n}\right) & =\min \left\{\alpha_{3,1} c_{1}, c_{3}\right\}, \\
f\left(\rho_{1, N x_{1}}^{n}, t^{n}\right) & =f\left(\rho_{2,0}^{n}, t^{n}\right)+f\left(\rho_{3,0}^{n}, t^{n}\right),
\end{aligned}
$$

with

$$
c_{1}=D_{1}\left(\rho_{1, N x_{1}-0.5}^{n}, t^{n}\right), \quad c_{2}=S_{2}\left(\rho_{2,0.5}^{n}, t^{n}\right), \quad c_{3}=S_{3}\left(\rho_{3,0.5}^{n}, t^{n}\right) .
$$

For junctions with two incoming roads $e=1$ and $e=2$, one outgoing road $e=3$, and priority parameter $P \in(0,1)$, we use (according to (4))

$$
\begin{aligned}
f\left(\rho_{1, N x_{1}}^{n}, t^{n}\right) & =\min \left\{c_{1}, \max \left\{P c_{3}, c_{3}-c_{2}\right\}\right\}, \\
f\left(\rho_{2, N x_{2}}^{n}, t^{n}\right) & =\min \left\{c_{2}, \max \left\{(1-P) c_{3}, c_{3}-c_{1}\right\}\right\}, \\
f\left(\rho_{3,0}^{n}, t^{n}\right) & =f\left(\rho_{1, N x_{1}}^{n}, t^{n}\right)+f\left(\rho_{2, N x_{2}}^{n}, t^{n}\right),
\end{aligned}
$$

with

$$
c_{1}=D_{1}\left(\rho_{1, N x_{1}-0.5}^{n}, t^{n}\right), \quad c_{2}=D_{2}\left(\rho_{2, N x_{2}-0.5}^{n}, t^{n}\right), \quad c_{3}=S_{3}\left(\rho_{3,0.5}^{n}, t^{n}\right) .
$$

Note that $c_{2}=D_{2}^{c}\left(l_{2}^{n}, t^{n}\right)$ in the case of a controlled on-ramp junction as described in (13) and (14).

\section{Inflow conditions}

According to (5), we apply

$$
f\left(\rho_{e, 0}^{n}, t^{n}\right)=\min \left\{f_{e}^{\text {in }}\left(t^{n}\right), S_{e}\left(\rho_{e, 0.5}^{n}, t^{n}\right)\right\}
$$

at an inflow boundary with a desired inflow rate $f_{e}^{\text {in }}\left(t^{n}\right)$. In the presence of a queue, according to (6), (7), (8) and (13), we use

$$
f\left(\rho_{e, 0}^{n}, t^{n}\right)=\min \left\{c_{\text {queue }}, S_{e}\left(\rho_{e, 0.5}^{n}, t^{n}\right)\right\}
$$

with

$$
c_{\text {queue }}=w_{e}\left(t^{n}\right) \min \left\{f_{e}^{\text {in }}\left(t^{n}\right)+\frac{l_{e}^{n}}{\Delta t}, \tilde{f}_{e}^{\max }\right\},
$$

and the explicit Euler scheme

$$
l_{e}^{n+1}=l_{e}^{n}+\Delta t\left(f_{e}^{\text {in }}\left(t^{n}\right)-f\left(\rho_{e, 0}^{n}, t^{n}\right)\right),
$$

where $l_{e}^{n} \approx l_{e}\left(t^{n}\right)$.

At an outflow boundary at edge $e$ with maximum outflow rate $f_{e}^{\text {out }}\left(t^{n}\right)$, we use (see (9))

$$
f\left(\rho_{e, N x}^{n}, t^{n}\right)=\min \left\{f_{e}^{\text {out }}\left(t^{n}\right), D_{e}\left(\rho_{e, N x-0.5}^{n}, t^{n}\right)\right\} .
$$




\section{Entire Simulation Procedure}

For given initial conditions for the traffic flow network and prescribed speed limits and ramp controls, the presented discretizations allow for an approximate solution of our model. The basic steps of this procedure are given in Algorithm 1. Starting from the initial state at time $t^{0}=0$, one may iteratively compute the flow rates at the current time $t^{n}$ (for $n=0, \ldots, N t-1$ ), and subsequently the density values and queue lengths at the next time $t^{n+1}$.

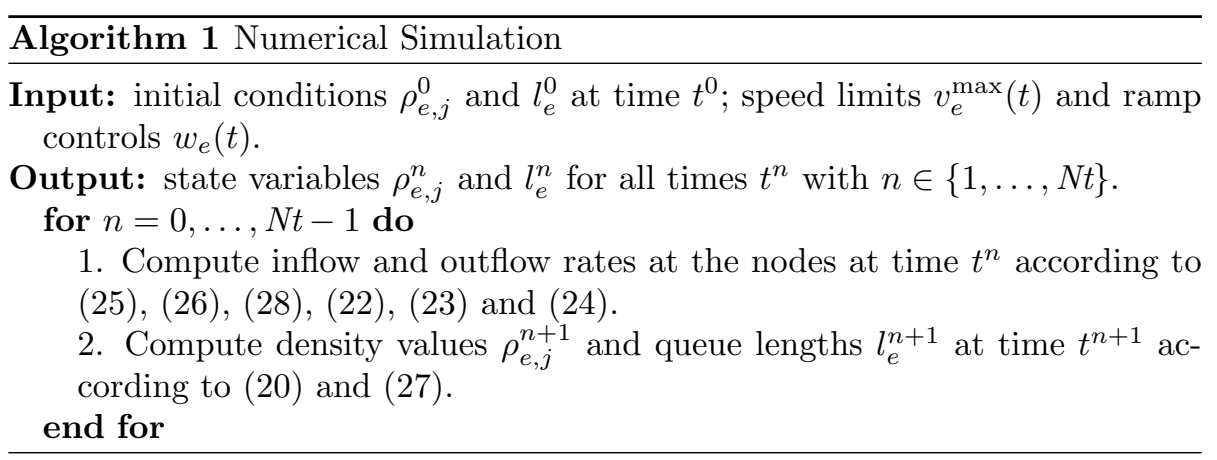

\subsection{Optimization approach}

Based on the presented discretization and the simulation procedure shown in Algorithm 1, we want to solve the underlying (finite dimensional) optimization task with an SQP solver (DONLP2) [29,30]. This necessitates the evaluation of the objective function and the computation of gradient information. The (discretized) objective function (18) can be directly evaluated after running a simulation according to Algorithm 1. For the necessary gradient information we apply a first-discretize adjoint approach. The main steps of the underlying procedure are given in Algorithm 2.

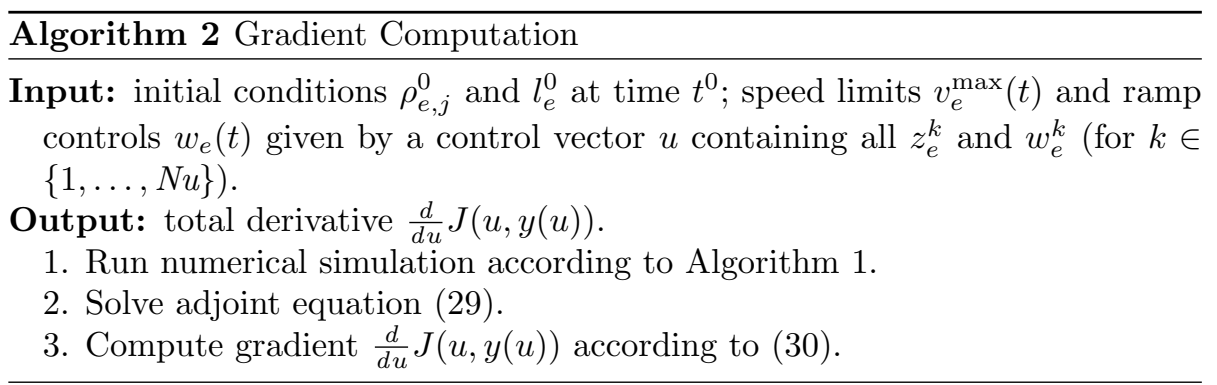

To describe the applied adjoint approach, we consider the objective function $J(u, y)$ (in our case given by (18)), where $u$ contains all control variables of the discretized model equations (the speed limits $z_{e}^{k}$ and the ramp controls $w_{e}^{k}$ ) and $y$ all state variables (densities $\rho_{e}^{n}$, flow rates $f\left(\rho_{e}^{n}\right)$, queue lengths $\left.l_{e}^{n}\right)$. Further, we summarize all discretized model equations, or constraints respectively, as $E(u, y)=0$. Assuming that for given control variables $u$ the model equations $E(u, y)=0$ have a unique solution, one may consider $y=y(u)$ and the so-called 
reduced problem with the objective function $J(u)=J(u, y(u))$. Note that the solution of $E(u, y)=0$ corresponds to running Algorithm 1.

With the so-called adjoint state $\xi$ given by the solution of the adjoint equation

$$
\left(\frac{\partial}{\partial y} E(u, y(u))\right)^{T} \xi=-\left(\frac{\partial}{\partial y} J(u, y(u))\right)^{T},
$$

one can efficiently compute the cost gradient as

$$
\frac{d}{d u} J(u, y(u))=\frac{\partial}{\partial u} J(u, y(u))+\xi^{T} \frac{\partial}{\partial u} E(u, y(u)) .
$$

In fact, the partial derivatives of $J$ and $E$ with respect to $u$ and $y$ can be easily computed and (29) is a single linear system with a special structure that can be easily exploited (see for instance $[20,21]$ ). We remark that some of the discretized model equations are continuous but not differentiable with respect to $u$ and $y$ so that smoothing is necessary. Incidentally, we also remark that the solution given by the gradient-based descent method is only locally optimal.

As already noted above, the time points $t^{n}$ of the discretization may differ from the control points $\nu^{k}$. The values of the controls $v_{e}^{\max }(t)$ and $w_{e}(t)$ at $t=t^{n}$, which are needed within Algorithms 1 and 2, are computed according to (12) and (16). Regarding the computation of gradient information using Algorithm 2, this necessitates the application of the chain rule (cf. again [20]), where in our case the "inner derivatives" equal zero or one due to the piecewise constant control functions.

\section{Numerical Results}

This section collects the numerical results corresponding to three different scenarios. The first example is concerned with variable speed limits to control traffic flow on a road network similar to the Frankfurter Kreuz, which is a famous German Autobahn interchange. Here we vary discretization as well as control grid parameters to study their influence on the optimal solution. In the second example we consider the combined optimization of variable speed limits (VSL) and ramp metering. The topology of the third example is quite similar to the second one, but refers to a real world case study.

\subsection{Variable Speed Limits}

Inspired by the Autobahn interchange in Frankfurt, Germany, we present a corresponding topology of the road network (including distribution rates $\alpha$ and priority parameters $P$ ) in Figure 5. Further road properties as well as the initial conditions are given in Table 1. Additionally, Figure 6 shows the inflow profiles within the considered time horizon of 1000 seconds.

For the described setting we consider the following experiments:

1. Optimization for a fixed discretization $(\Delta x=50, \Delta t=0.5$, Lax-Friedrichs scheme) and various numbers of control points $N u$.

2. Optimization for a fixed number of control points $(N u=20)$ and various discretization parameters. 


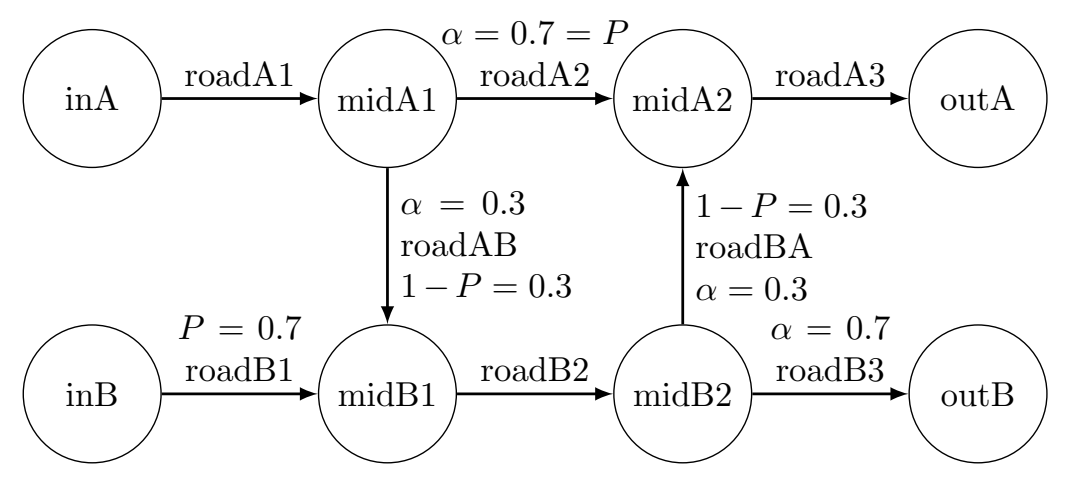

Figure 5: Road network with two main roads.

Table 1: Properties of the roads in Figure 5

\begin{tabular}{c|c|c|c|c|c} 
road & length & $\rho_{\max }$ & $v_{\text {low }}$ & $v_{\text {high }}$ & initial density \\
\hline \hline roadA1 & 1000 & 2 & 20 & 30 & 0.3 \\
roadA2 & 1000 & 2 & 20 & 30 & 0.3 \\
roadA3 & 1000 & 2 & 20 & 30 & 0.3 \\
roadB1 & 1000 & 2 & 20 & 30 & 0.3 \\
roadB2 & 1000 & 2 & 20 & 30 & 1.2 \\
roadB3 & 1000 & 2 & 20 & 30 & 1.2 \\
roadAB & 200 & 0.5 & 10 & 20 & 0.1 \\
roadBA & 200 & 0.5 & 10 & 20 & 0.1
\end{tabular}

3. We finally consider the same experiment as in 1 . with a fixed discretization but an additional penalty term with weight $\delta_{e} \geq 0$ in (18):

$$
\sum_{e} \delta_{e} \sum_{n=1}^{N t} \Delta t\left(\frac{v_{e}^{\max }\left(t^{n}\right)-v_{e}^{\max }\left(t^{n-1}\right)}{v_{e}^{\text {high }} \Delta t}\right)^{2}
$$

in order to get smoother controls. This is similar to [15].

Table 2 and Figure 7 show the results for the first test. While the discretization parameters are kept fixed, we consider an increasing number of control points $N u$ (starting with $N u=5$ in the first line of Figure 7 until $N u=80$ in the last line) and run the optimization once for minimizing the total travel time (with $\beta_{e}=10^{-3}$ ) and once for maximizing the accumulated outflow at the nodes outA and outB (with $\varepsilon_{e}=10^{-1}$ ). As expected one gets lower travel times / larger outflows for an increasing number of control points. In particular, compared to the uncontrolled case, where all speed limits are taken at the upper bound, we achieve an improvement of $1.28 \%$ / 0.03\%. Exemplarily, Figure 8 compares the traffic density in the middle of roadB2 with and without optimization of speed limits (with respect to the total travel time). The computing time for a single simulation with the given discretization parameters is roughly one second on an Intel(R) Core(TM) i5-3340M CPU with 2.70GHz.

In the second experiment we vary the discretization parameters and keep the number of control points constant for the minimization of the total travel time / maximization of the outflow. Table 3 and Figure 9 show the results 


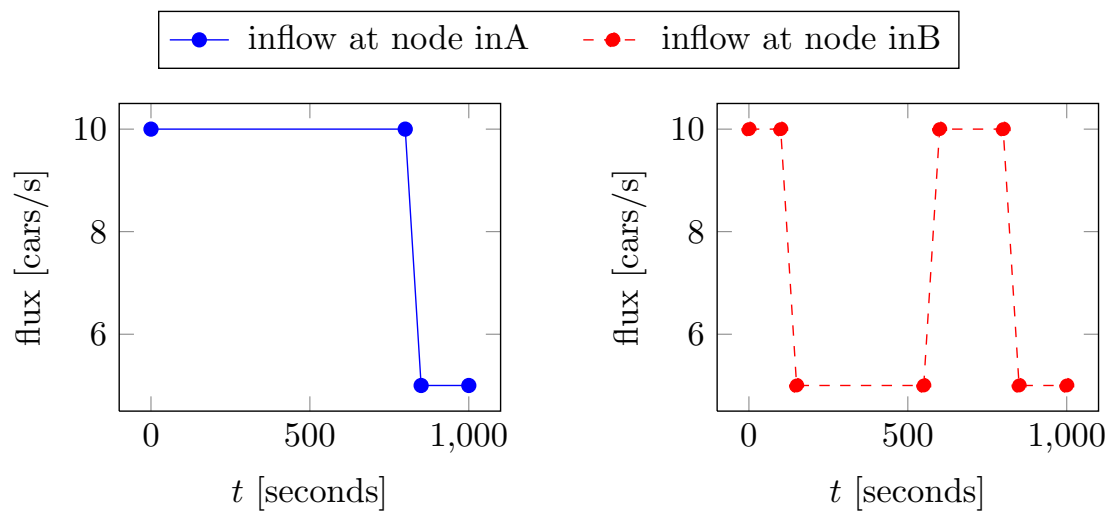

Figure 6: Inflow profiles for the network in Figure 5.

Table 2: Optimal travel times / outflows for different numbers of control points

\begin{tabular}{c|c|c|c}
$\mathrm{Nu}$ & $\Delta x / \Delta t$ & min. travel time & max. outflow \\
\hline \hline- & $50 / 0.5$ & 2336.698921 & 1844.762416 \\
5 & $50 / 0.5$ & 2307.370375 & 1845.023309 \\
10 & $50 / 0.5$ & 2307.157634 & 1845.030866 \\
20 & $50 / 0.5$ & 2306.863796 & 1845.289234 \\
40 & $50 / 0.5$ & 2306.778537 & 1845.290014 \\
80 & $50 / 0.5$ & 2306.694086 & 1845.353672
\end{tabular}

for these computations (with $\Delta x=200$ in the first line of Figure 9 down to $\Delta x=12.5$ in the last line). Note that the presented values of the objective function (scaled travel times / outflows) are evaluated using a simulation with the finest discretization parameters.

Table 3: Optimal travel times / outflows for different discretizations

\begin{tabular}{c|c|c|c}
$\mathrm{Nu}$ & $\Delta x$ (max.) $/ \Delta t$ & min. travel time & max. outflow \\
\hline \hline- & - & 2330.987519 & 1846.918574 \\
20 & $200 / 0.500$ & 2369.960626 & 1825.869588 \\
20 & $100 / 0.500$ & 2333.714043 & 1836.601771 \\
20 & $50 / 0.500$ & 2306.708163 & 1845.327369 \\
20 & $25 / 0.250$ & 2300.563136 & 1846.649973 \\
20 & $12.5 / 0.125$ & 2297.448274 & 1847.150023
\end{tabular}

Similar to above, the finer the discretization parameters are, the better the computed optimal solutions are. For the (too) coarse discretizations, one actually gets "optimal controls", which are worse than the "no control" case (all speed limits at the upper bound). Note that this may only happen because we finally evaluate the computed controls by a simulation using the finest grid.

The results of the third experiment are shown in Figure 10. Obviously, the additional penalty term, which penalizes variations in the control, leads to smoother optimal solutions. 


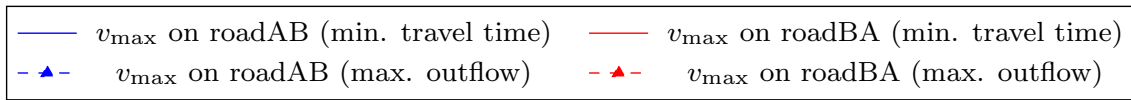
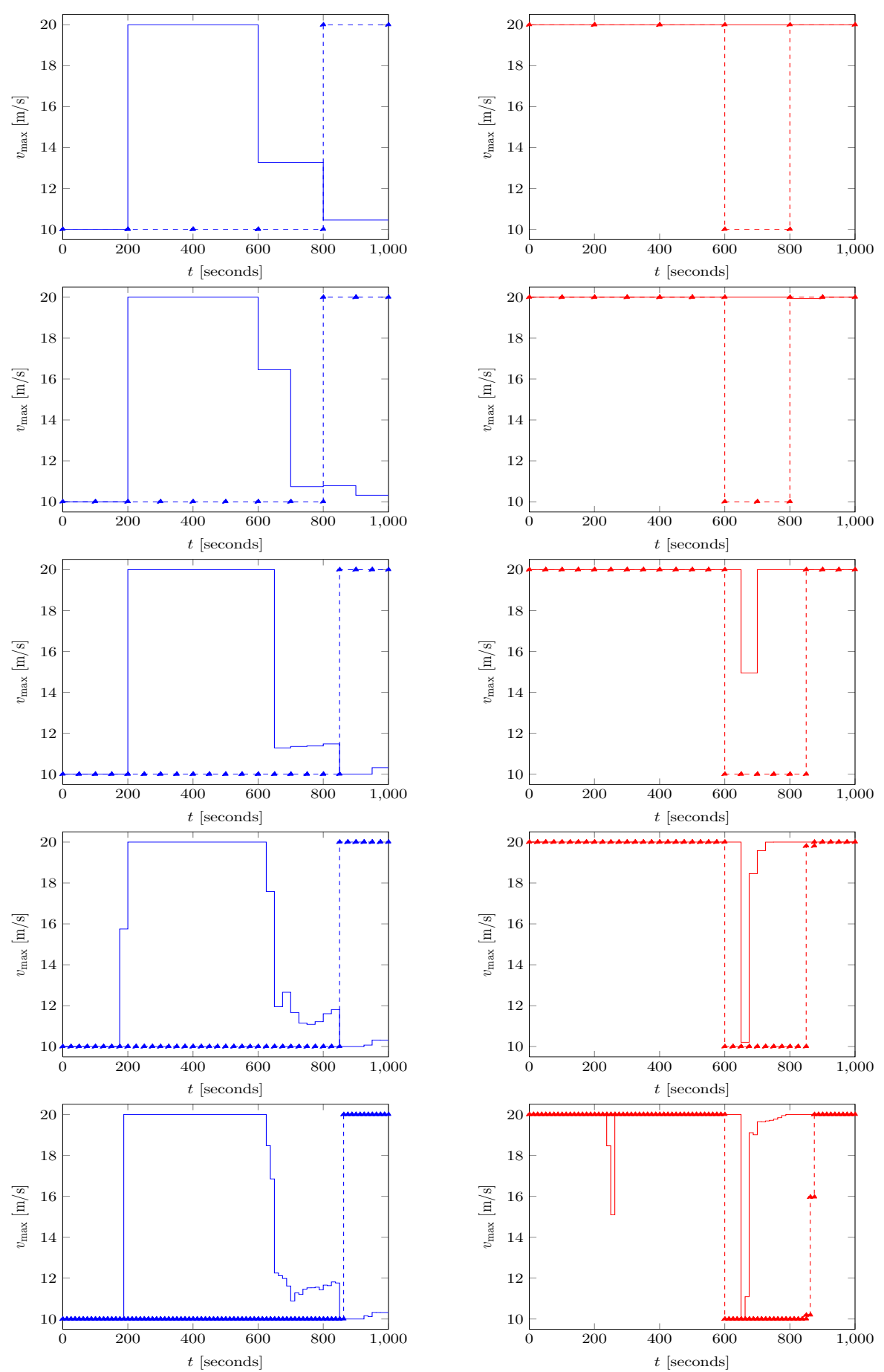

Figure 7: Optimal control of $v_{\max }$ for an increasing number of control points. 


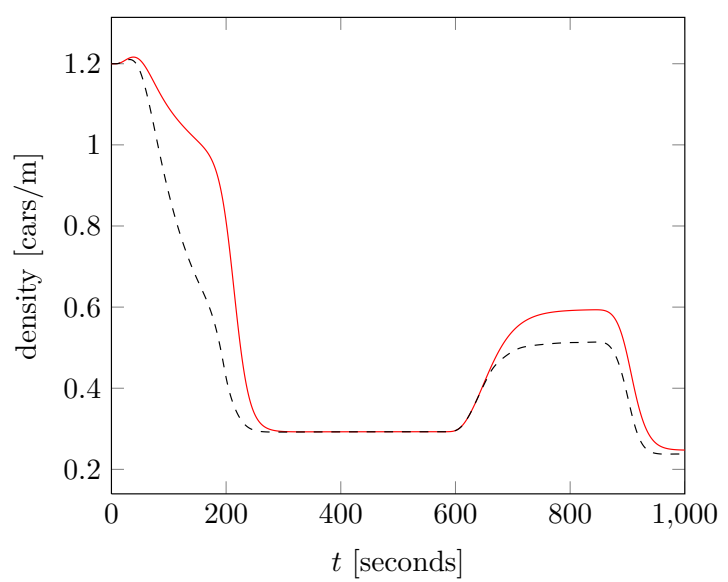

Figure 8: Density on roadB2 with and without optimization of speed limits.

\subsection{VSL meets Coordinated Ramp Metering}

In our second example, we consider the combined optimization of variable speed limits and ramp metering. The topology of the underlying network is shown in Figure 11 and the corresponding parameters are given in Table 4 . The priority parameter at the on-ramp is $P=0.5$ and we take $f^{\max }=1.5$. Figure 12 shows the inflow profiles and the maximum outflow. Again, we consider several discretizations and also compare the results of the Lax-Friedrichs scheme with the Godunov scheme. Depending on the chosen resolution, a single simulation for the considered time horizon of 3 hours takes between $0.1 \mathrm{~s}$ ( $\Delta t=30$ and $\Delta x=1000)$ and about $13 \mathrm{~s}(\Delta t=0.75$ and $\Delta x=25)$. For the optimization we use $N u=36$ control points and consider the congestion measure (19) subject to the following constraints: We allow a maximum size of the queue at the inflow of the main road (node "in") of 50 cars and a maximum size of the queue at the on-ramp of 600 cars. Both constraints are violated without optimization $\left(v_{\max }\right.$ at the upper bound and $w(t)=1$ at the on-ramp). Note that the gradients of these constraints, which are necessary for the applied SQP solver, are computed exactly in the same manner as the gradients of the objective (as described in Section 3.2).

Table 4: Properties of the roads in Figure 11

\begin{tabular}{c|c|c|c|c|c} 
road & length & $\rho_{\max }$ & $v_{\text {low }}$ & $v_{\text {high }}$ & initial density \\
\hline \hline road1a & 2000 & 2 & 30 & 30 & 0.1 \\
road1b & 2000 & 2 & 10 & 30 & 0.1 \\
road2 & 4000 & 2 & 30 & 30 & 0.1
\end{tabular}

Figure 13 shows the inflow to the main road from the on-ramp in the uncontrolled case, nicely validating the different situations that may occur at an on-ramp: Initially the full inflow goes to the main road $\left(f^{\text {in }}=0.75\right)$. Due to the congestion at road 2 , the inflow drops down to $P \cdot f^{\text {out }}=0.5$ (outflow at the 


\begin{tabular}{|c|c|c|c|}
\hline & $v_{\max }$ on roadAB (min. travel time) & & $v_{\max }$ on roadBA (min. travel time) \\
\hline$-\rightarrow-$ & $v_{\max }$ on $\operatorname{roadAB}(\max$. ol & $-\Delta-$ & $v_{\max }$ on roadBA (max. outflow) \\
\hline
\end{tabular}
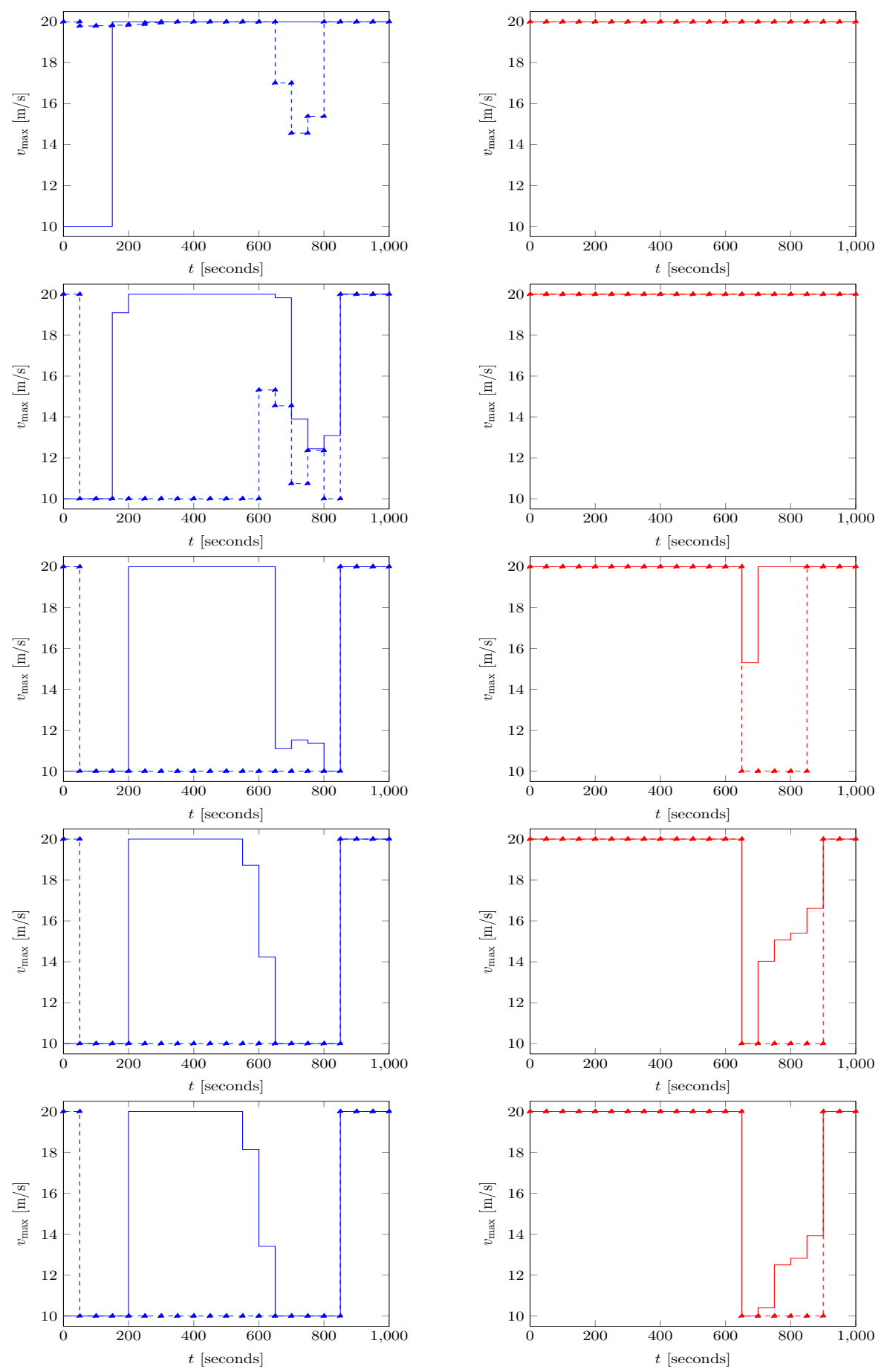

Figure 9: Optimal control of $v_{\max }$ for different discretizations. 


\begin{tabular}{|ccc|}
\hline & $v_{\max }$ on roadAB & $v_{\max }$ on roadBA \\
$-{ }_{-}$ & $v_{\max }$ on roadAB $($ with penalty term) & $-v_{\max }$ on roadBA (with penalty term) \\
\hline
\end{tabular}
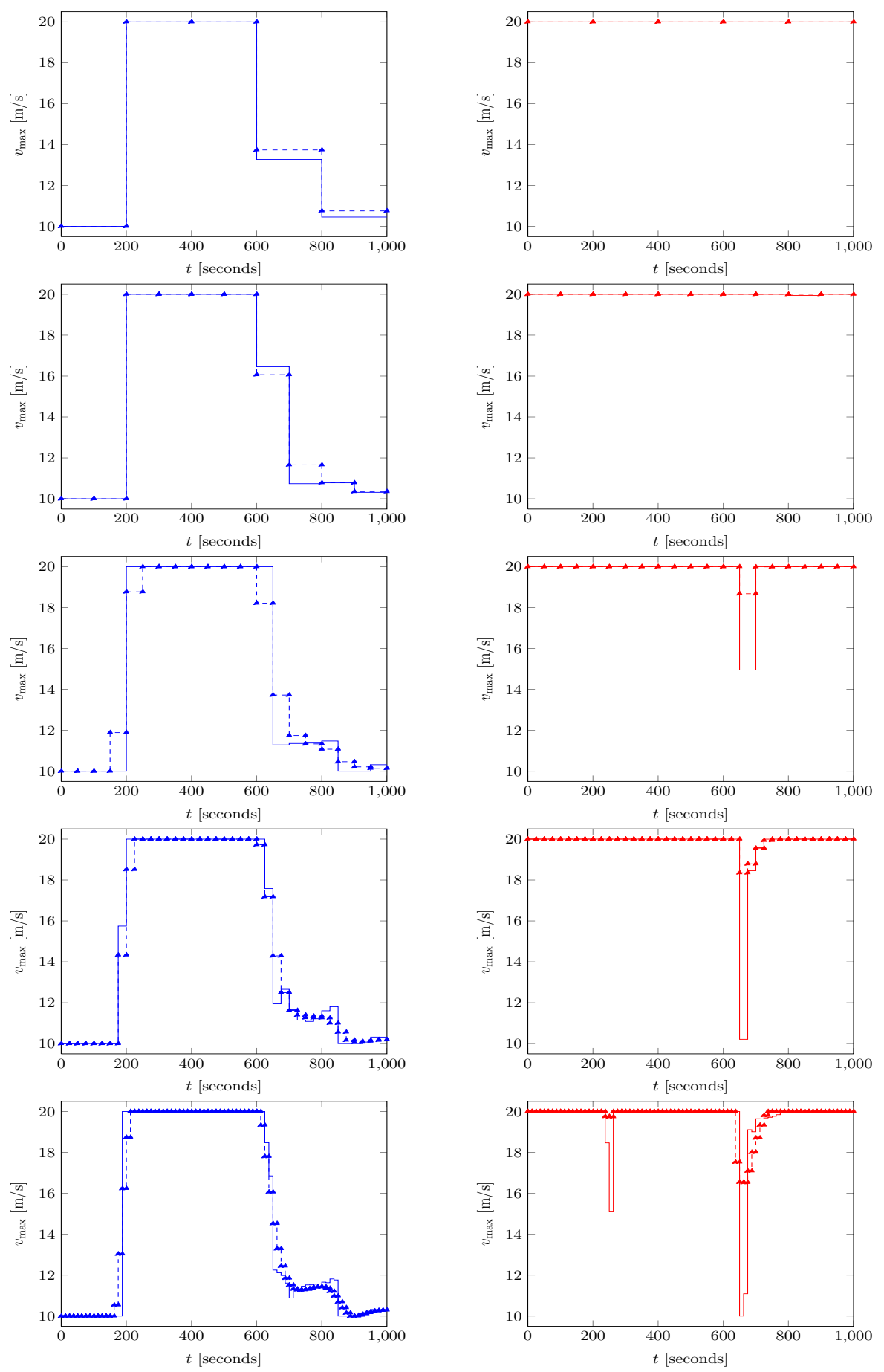

Figure 10: Optimal control of $v_{\max }$ with and without penalty term (min. travel time). 


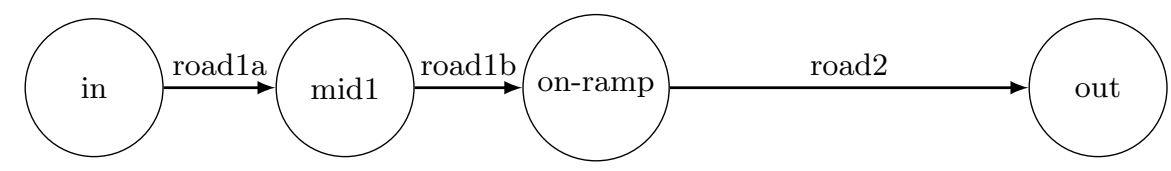

Figure 11: Road network with an on-ramp at the node "on-ramp".

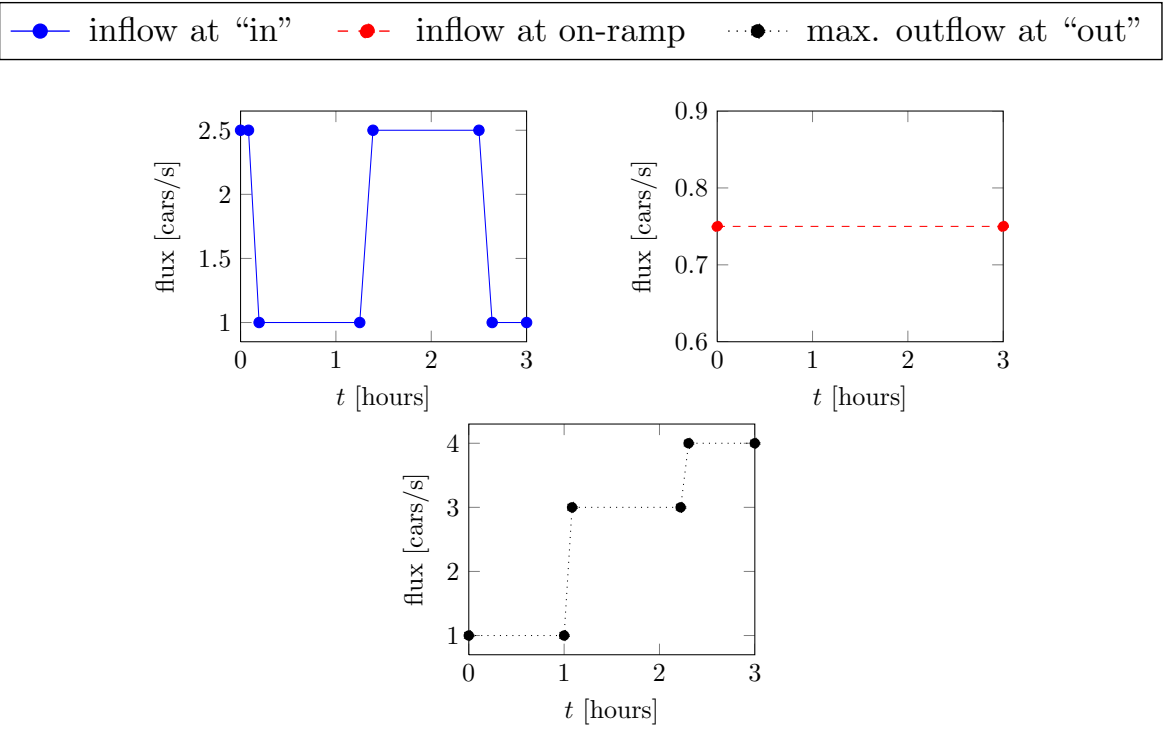

Figure 12: Inflow/max. outflow profiles for the network in Figure 11.

node "out" is 1.0 and priority parameter $P=0.5$ ). When the maximum possible outflow at the node "out" increases, also the flow on the on-ramp increases up the maximum flow level $\left(\tilde{f}^{\max }=1.5\right)$. When the queue at the on-ramp is empty, the inflow to the main road from the on-ramp decreases to the inflow at this node again $\left(f^{\text {in }}=0.75\right)$.

Table 5 shows the results of the optimization procedure. The first (four) column(s) contain the parameters used for the optimization (number of control points, discretization parameters, applied scheme). The resulting controls have then been evaluated by simulations with the same scheme and discretization parameters, but also with a "reference discretization" $(\Delta t=0.75, \Delta x=25$, Godunov scheme). The last column shows the improvement in the computed objective function - always comparing the "optimal" solution with the uncontrolled case for simulations with the same discretization parameters and the same scheme. Obviously, the considered discretization has a strong impact on the improvement. On the one hand, let us first consider the evaluations with the reference discretization: Even the optimal solution found with the coarsest discretization of the Lax-Friedrichs scheme $(\Delta x=1000)$ yields only slightly worse results than the best solution found (1.58\% towards $1.75 \%)$. On the other hand, when comparing the uncontrolled and the optimized case with the coarsest discretization of the Lax-Friedrichs scheme, one gets an improvement of $12.22 \%$. This effect certainly has to be taken into account, when comparing optimization results for a well-resolved PDE model with other existing approaches: In 
the benchmark problem in [14] for instance, the discretization parameters are $\Delta x=1000$ (metres) and $\Delta t=10$ (seconds).

Table 5: Optimization results and actual improvement for various discretization parameters

\begin{tabular}{|c|c|c|c|c|c|c|c|c|}
\hline \multicolumn{4}{|c|}{ optimization } & \multicolumn{3}{|c|}{ simulation } & \multirow[t]{2}{*}{ objective } & \multirow[t]{2}{*}{ improvement } \\
\hline$N u$ & $\Delta x$ & $\Delta t$ & scheme & $\Delta x$ & $\Delta t$ & scheme & & \\
\hline \multirow[t]{5}{*}{ "0 } & & & & 1000 & 15.00 & LF & 19.771085 & - \\
\hline & & & & 1000 & 30.00 & $\mathrm{G}$ & 20.414095 & - \\
\hline & & & & 100 & 1.50 & $\mathrm{LF}$ & 21.056753 & - \\
\hline & & & & 100 & 3.00 & $\mathrm{G}$ & 21.338182 & - \\
\hline & & & & 25 & 0.75 & G & 21.422205 & - \\
\hline \multirow[t]{2}{*}{36} & 1000 & 15.00 & $\overline{\mathrm{LF}}$ & 1000 & 15.00 & $\overline{\mathrm{LF}}$ & 17.354620 & $12.22 \%$ \\
\hline & & & & 25 & 0.75 & $\mathrm{G}$ & 21.082987 & $1.58 \%$ \\
\hline \multirow[t]{2}{*}{36} & 1000 & 30.00 & $\mathrm{G}$ & 1000 & 30.00 & $\mathrm{G}$ & 18.985271 & $7.00 \%$ \\
\hline & & & & 25 & 0.75 & $\mathrm{G}$ & 21.063006 & $1.68 \%$ \\
\hline \multirow[t]{2}{*}{36} & 100 & 1.50 & $\mathrm{LF}$ & 100 & 1.50 & $\mathrm{LF}$ & 20.437825 & $2.94 \%$ \\
\hline & & & & 25 & 0.75 & $\mathrm{G}$ & 21.081400 & $1.59 \%$ \\
\hline \multirow[t]{2}{*}{36} & 100 & 3.00 & $\mathrm{G}$ & 100 & 3.00 & G & 20.886909 & $2.11 \%$ \\
\hline & & & & 25 & 0.75 & G & 21.047366 & $1.75 \%$ \\
\hline
\end{tabular}

Finally, Figures 14, 15 and 16 show the computed optimal controls for the finest discretization and the Godunov scheme, the queue lengths at the inflow of the main road and at the on-ramp in the uncontrolled and the optimized cases, and the density at the beginning of road2, respectively. The speed control is in particular active during the times of large inflows (at the beginning and after approximately one and a half hour), while the on-ramp control takes care of the bound on the queue. Further, in the optimal solution, the queue at the beginning of the road stays empty, whereas there is a new large peak in the queue at the on-ramp during the high traffic time after about 2 hours because of the reduced density on the main road.

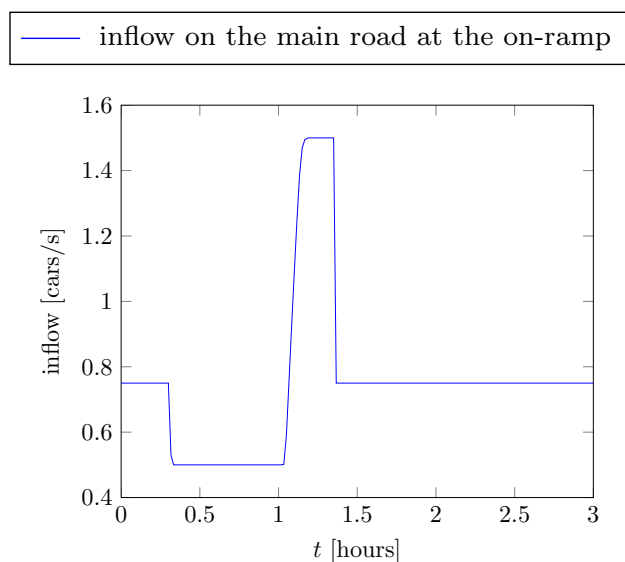

Figure 13: Inflow on the main road at the on-ramp without optimization. 


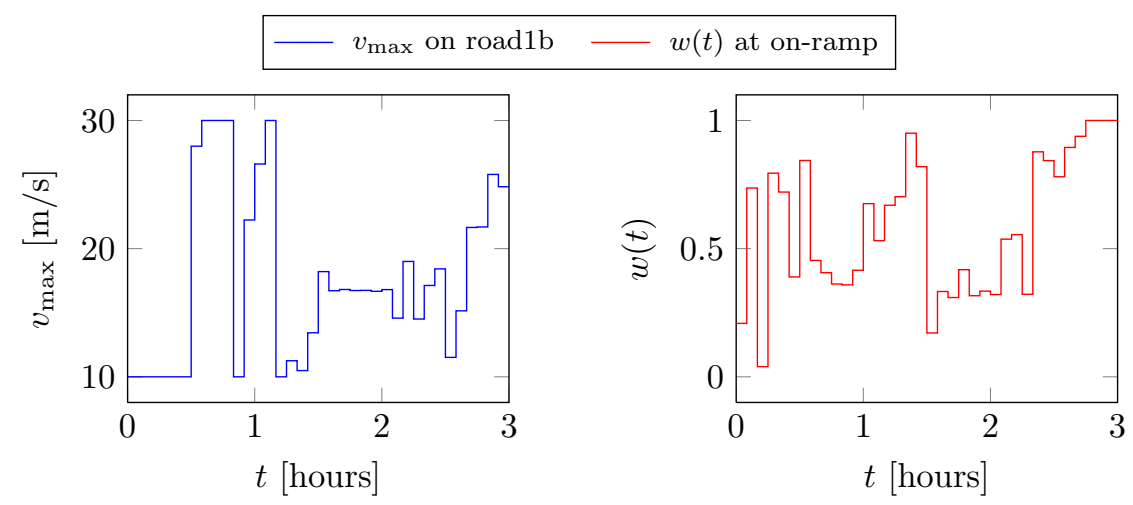

Figure 14: Optimal control of $v_{\max }(t)$ on road1b and $w(t)$ at the on-ramp.

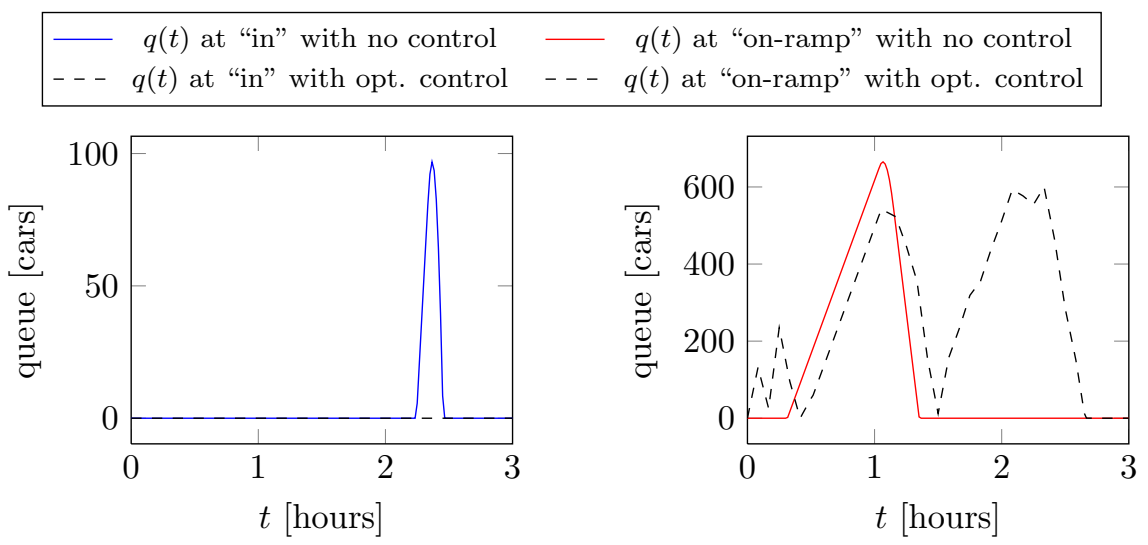

Figure 15: Queue at the node "in" and the on-ramp with and without optimization. 


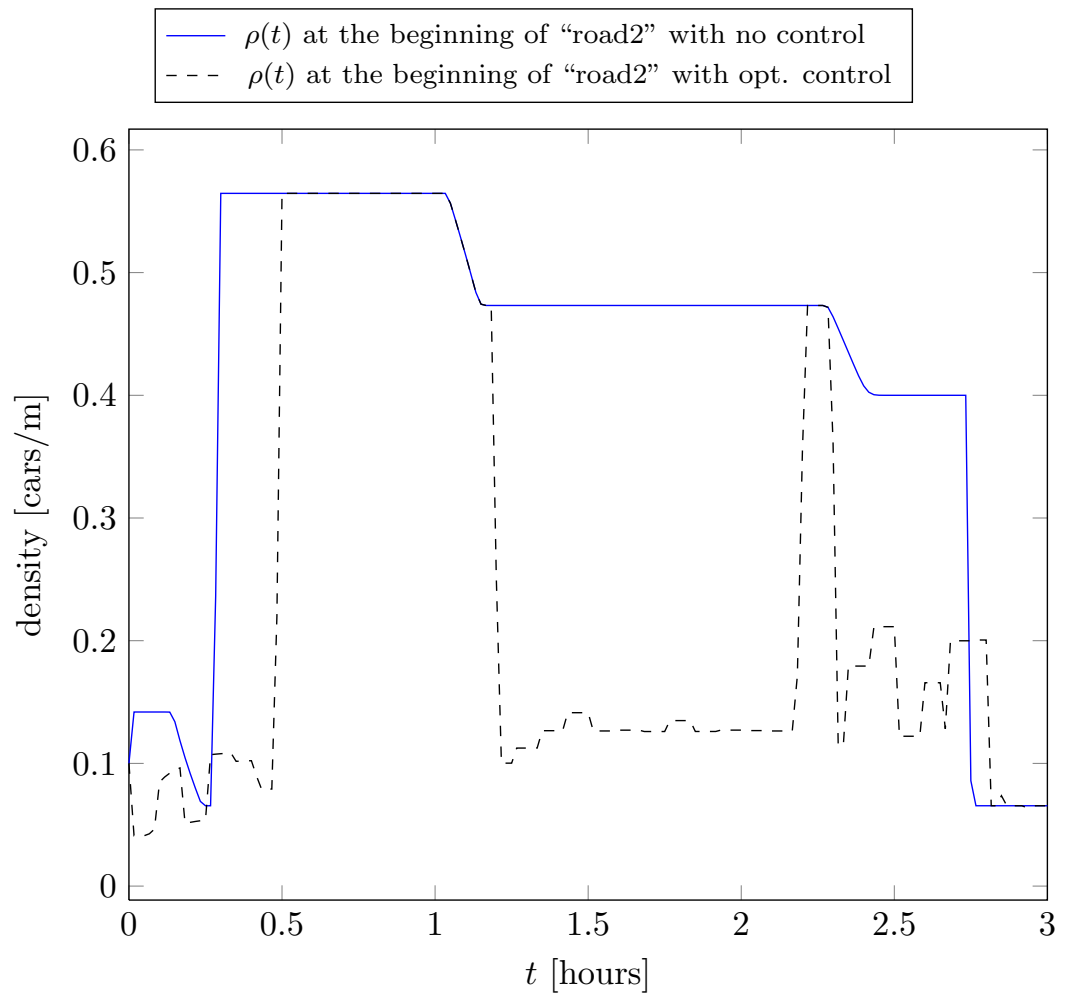

Figure 16: Density at the beginning of road2 with and without optimization. 


\subsection{Real World Example: From Antibes to Nice}

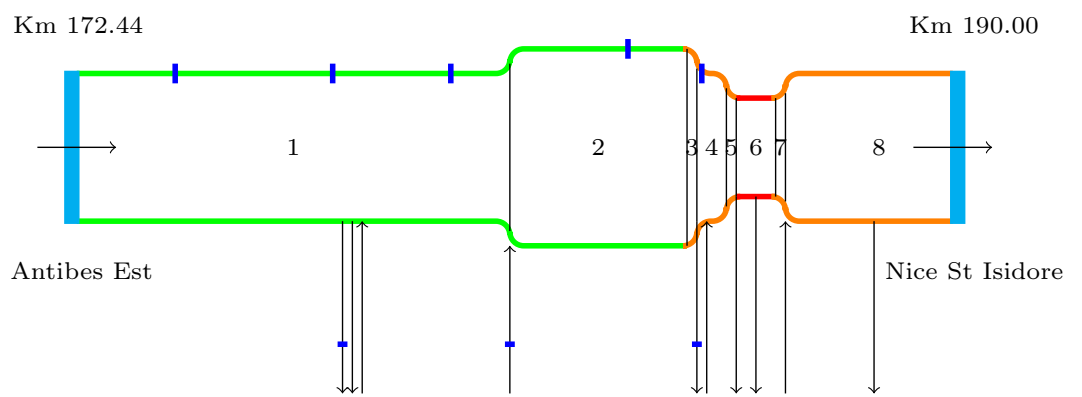

Figure 17: (Taken from [3].) A8 highway's scheme between Antibes and Nice St. Isidore. Blue spots indicate loop detectors' locations. Light blue lines indicate toll stations' locations. Black arrows indicate the direction of cars flow, where those on the bottom are on and off ramps. Highway's sections limited by green lines are those where the maximal speed limit is $110 \mathrm{~km} / \mathrm{h}$, while in the orange ones is 90 and in the red one is $70 \mathrm{~km} / \mathrm{h}$. Width is proportional to the number of lanes: 2, 3 or 4 . For further details see [3].

Numerical simulations performed in this section correspond to sections 1 to 4 .

Inspired by the real network depicted in Figure 17, we consider a stretch of highway of $9 \mathrm{~km}$ long divided in cells of $\Delta x=100 \mathrm{~m}$ each as follows:

- a section of $5 \mathrm{~km}$ between $x_{0}=0 \mathrm{~km}$ and $x_{1}=5 \mathrm{~km}$ consisting of 3 lanes $\left(\rho_{1}^{\max }=450\right.$ cars $\left./ \mathrm{km}\right)$ with maximal speed $v_{1}^{\max }=110 \mathrm{~km} / \mathrm{h}$;

- a section of $3 \mathrm{~km}$ between $x_{1}=5 \mathrm{~km}$ and $x_{2}=8 \mathrm{~km}$ consisting of 4 lanes $\left(\rho_{2}^{\max }=600\right.$ cars $\left./ \mathrm{km}\right)$ with maximal speed $v_{2}^{\max }=110 \mathrm{~km} / \mathrm{h}$;

- a section of $1 \mathrm{~km}$ between $x_{2}=8 \mathrm{~km}$ and $x_{3}=9 \mathrm{~km}$ consisting of 3 lanes $\left(\rho_{3}^{\max }=450\right.$ cars $\left./ \mathrm{km}\right)$ with maximal speed $v_{3}^{\max }=90 \mathrm{~km} / \mathrm{h}$.

Moreover, an on-ramp link is connected to the mainline at the node $x_{1}$ (with priority parameter $P=0.25)$. We know that traffic is hindered downstream, that is, we impose a maximum outflow at $x_{3}$. The inflow and maximum outflow values are given in Figure 18. The computing time for a single simulation with $\Delta t=3$ seconds and the Godunov scheme for a 5 hour time horizon is roughly 2.5 seconds.

The speed limits $v_{1}^{\max }, v_{2}^{\max }$ and $v_{3}^{\max }$ are imposed by the authorities. We are interested in comparing the current situation resulting from the above choice of the parameters with the controlled one. We use the current speed limits as upper bound for the speed controls and $50 \mathrm{~km} / \mathrm{h}$ as lower bound for all roads.

The objective is again to minimize the congestion measure (19) subject to the following constraints:

- maximum size of the queue in Antibes: 50 cars,

- maximum size of the queue at the on-ramp: 100 cars. 


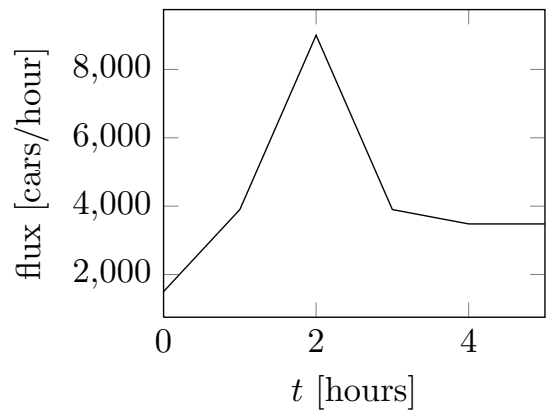

(a) Inflow at $x_{0}$.

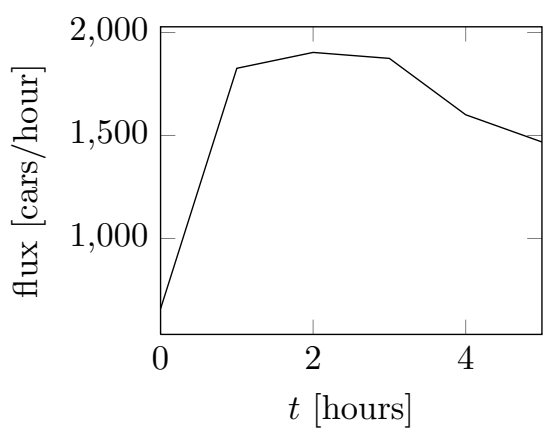

(c) Max. outflow at $x_{2}$ (off-ramp).

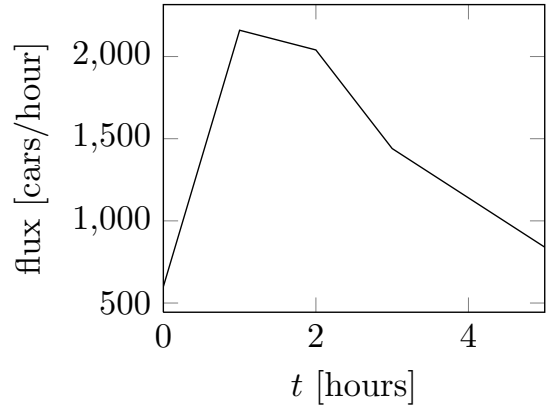

(b) Inflow at $x_{1}$ (on-ramp).

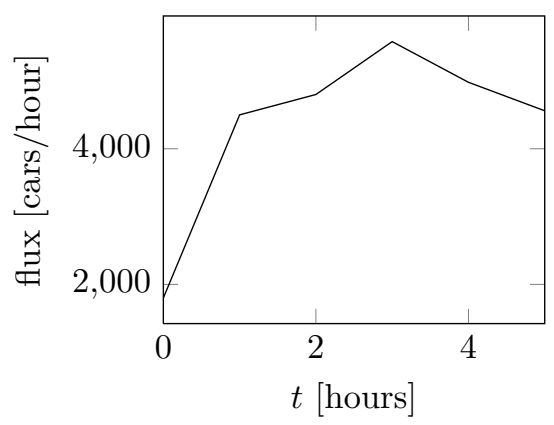

(d) Max. outflow at $x_{3}$.

Figure 18: Inflow/max. outflow profiles for considered network part of Figure 17.

Figure 19 shows the traffic density on the entire section at different times in the uncontrolled case. Due to the limited outflow, a traffic jam is building at (roughly) 7:15 (corresponding to $t=1.25$ hours, first plot), almost arriving at $x_{0}$ at about 8:30 (third plot) and having vanished approximately further 2 hours later.

Figure 20 shows the computed optimal controls $(N u=36)$. As a result, the congestion measure (19) could be reduced from 18.138 in the uncontrolled case to 17.222 , an improvement of roughly $5 \%$, which is of the same order as the results in [26]. In particular, the speed limit on road1 is (significantly) active for the first time during the peak time of the inflow (around $t=2$ ). The queues in the uncontrolled and the optimized case are plotted in Figure 21. Figure 22 shows the density at the beginning of road2 (behind the on-ramp). Similar to the results in Section 4.2, the price for the lower density on the main road is a further increase of the queue at the on-ramp.

\section{Conclusion}

In this work we have considered variable speed limits combined with coordinated ramp metering within the framework of the LWR network model. On one hand, the first order LWR model can be considered the most simple PDE model for traffic flow, which also allows for congestions. Nevertheless, the simulation 

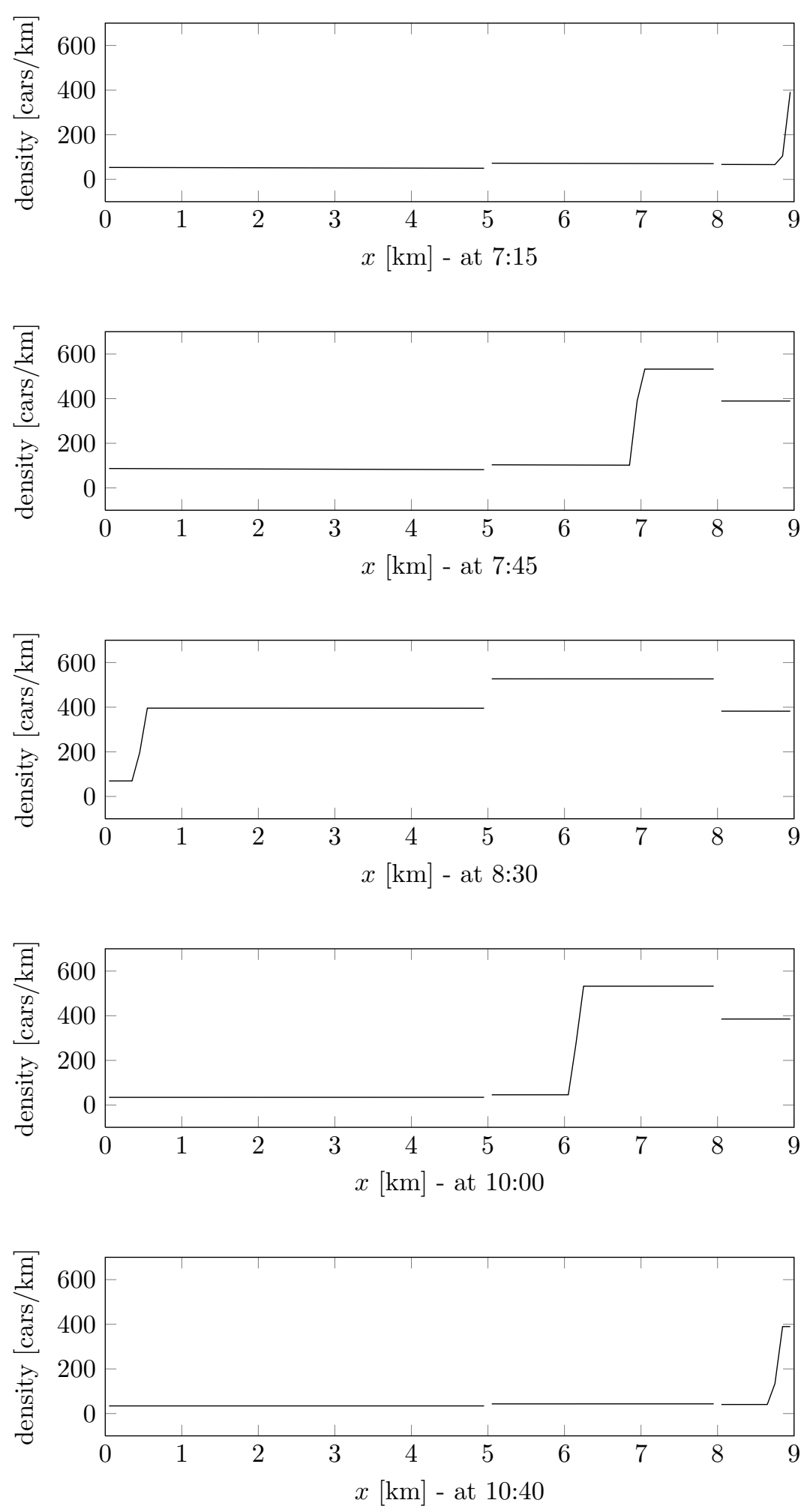

Figure 19: Traffic density between Antibes and Nice at different times. 


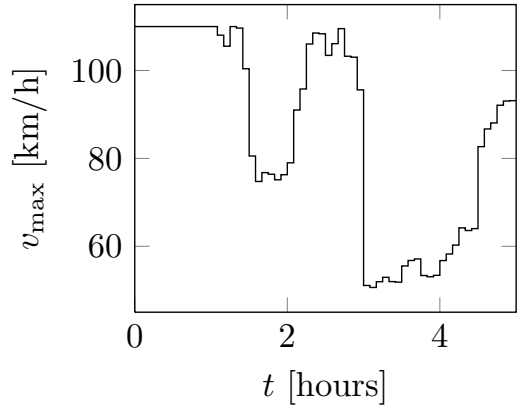

(a) road1

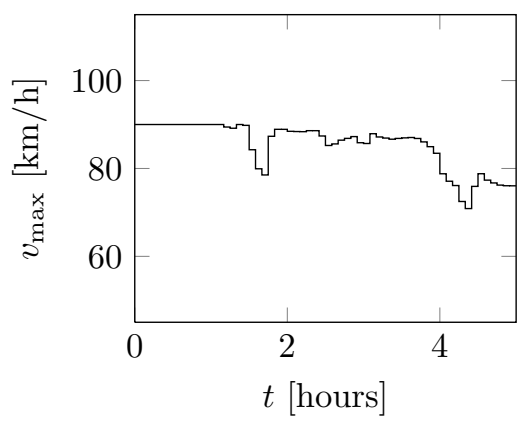

(c) $\operatorname{road} 3$

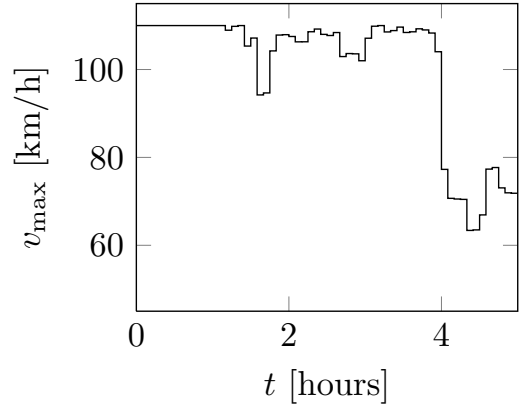

(b) road2

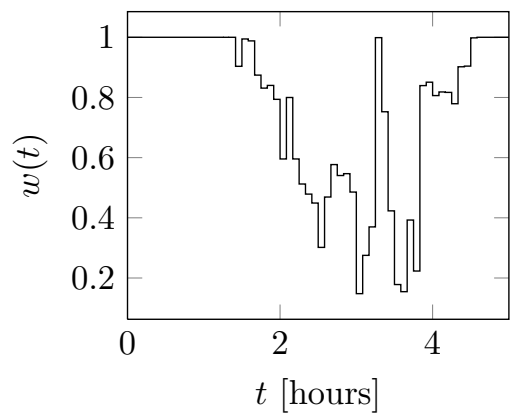

(d) on-ramp

Figure 20: Optimal control of $v_{\max }$ on road1, $\operatorname{road} 2$ and $\operatorname{road} 3$, and $w(t)$ at the on-ramp.

results for the real world example presented in Section 4.3 agreed well with the observed real situation. To improve the current situation, we applied a first discretize then optimize adjoint approach and sequential quadratic programming. While the computed optimal solutions for coarse discretizations often already delivered good results, we observed a strong dependence of the actual improvement with respect to the discretization parameters.

Further, the fulfillment of prescribed constraints was always successful within the presented framework. On the other hand, it seems to be a drawback of the applied first order model that within several scenarios one is not able to (significantly) improve the current situation in terms of total travel time or system outflow. This assumption is motivated by (additional) results we achieved for the scenario presented in Section 4.2, since for similar scenarios as in [14], where a "discrete second order model" but also a different model for the on-ramp flow are used, speed control and coordinated ramp metering also yield a significant improvement of the total travel time. Accordingly, in future work we are going to extend the presented framework to a second order PDE model and we also intend to consider different models for the on-ramp flow. 


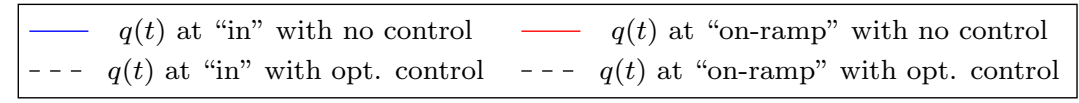
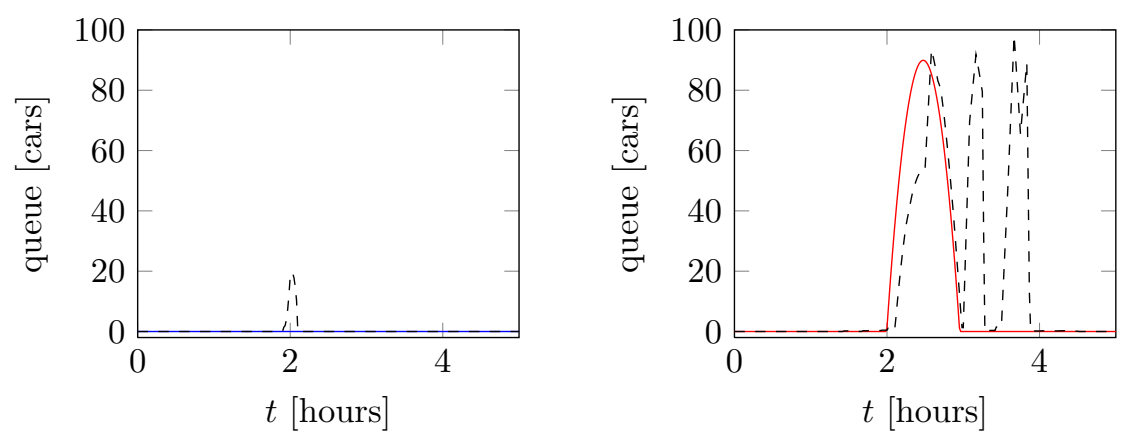

Figure 21: Queue at the node "in" and the on-ramp with and without optimization.

\section{Acknowledgments}

This work was financially supported by the project "Transport network modeling and analysis" co-funded by DAAD (Project-ID 57049018) and the PHC Procope (Project no. 30715QA).

\section{References}

[1] G. Bastin, B. Haut, J.-M. Coron, and B. D'Andréa-Novel, Lyapunov stability analysis of networks of scalar conservation laws, Netw. Heterog. Media, 2 (2007), pp. 751-759.

[2] G. Bretti, R. Natalini, and B. Piccoli, Numerical approximations of a traffic flow model on networks, Netw. Heterog. Media, 1 (2006), pp. 5784 .

[3] A. Cabassi and P. Goatin, Validation of traffic flow models on processed GPS data, Research Report RR-8382, 2013.

[4] R. C. Carlson, D. Manolis, I. Papamichail, and M. Papageorgiou, Integrated ramp metering and mainstream traffic flow control on freeways using variable speed limits, Procedia - Social and Behavioral Sciences, 48 (2012), pp. 1578 - 1588. Transport Research Arena 2012.

[5] G. M. Coclite, M. Garavello, and B. Piccoli, Traffic flow on a road network, SIAM J. Math. Anal., 36 (2005), pp. 1862-1886.

[6] G. M. Coclite And N. H. Risebro, Conservation laws with time dependent discontinuous coefficients, SIAM J. Math. Anal., 36 (2005), pp. 12931309. 


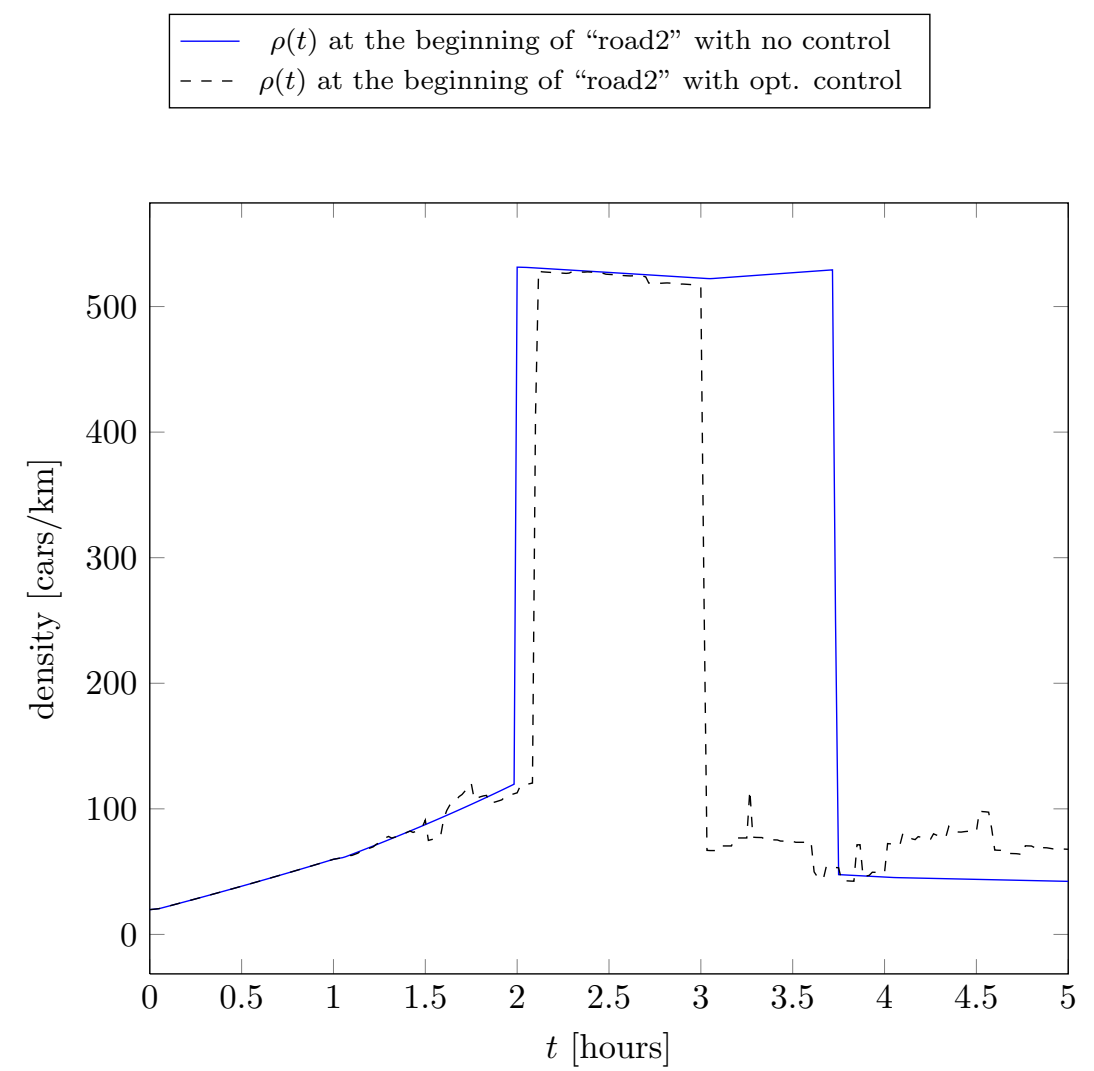

Figure 22: Density at the beginning of "road2" with and without optimization.

[7] A. Csikos, I. Varga, And K. M. Hangos, Freeway shockwave control using ramp metering and variable speed limits, 21st Mediterranean Conference on Control \& Automation, (2013), pp. 1569-1574.

[8] M. Garavello and B. Piccoli, Traffic flow on networks, Springfield, MO: American Institute of Mathematical Sciences (AIMS), 2006.

[9] - Conservation laws on complex networks, Ann. Inst. Henri Poincaré, Anal. Non Linéaire, 26 (2009), pp. 1925-1951.

[10] M. Garavello and B. Piccoli, Time-varying Riemann solvers for conservation laws on networks, J. Differential Equations, 247 (2009), pp. 447464 .

[11] M. B. Giles And N. A. Pierce, An introduction to the adjoint approach to design, Flow Turbul. Combust., 65 (2000), pp. 393-415.

[12] S. Göttlich and U. Zieglen, Traffic light control: a case study, Discrete Contin. Dyn. Syst., Ser. S, 7 (2014), pp. 483-501.

[13] M. Gugat, M. Herty, A. Klar, and G. Leugering, Optimal control for traffic flow networks, J. Optimization Theory Appl., 126 (2005), pp. 589-616. 
[14] A. Hegyi, B. D. Schutter, And H. Hellendoorn, Model predictive control for optimal coordination of ramp metering and variable speed limits, Transportation Research Part C, 13 (2005), pp. 185-209.

[15] A. Hegyi, B. D. Schutter, And J. Hellendoorn, Optimal coordination of variable speed limits to suppress shock waves, IEEE Transactions on Intelligent Transportation Systems, 6 (2005), pp. 102-112.

[16] M. HerTy AND A. Klar, Modeling, simulation, and optimization of traffic flow networks, SIAM J. Sci. Comput., 25 (2003), pp. 1066-1087.

[17] Simplified dynamics and optimization of large scale traffic networks, Math. Models Methods Appl. Sci., 14 (2004), pp. 579-601.

[18] H. Holden and N. H. Risebro, A mathematical model of traffic flow on a network of unidirectional roads, SIAM J. Math. Anal., 26 (1995), pp. 999-1017.

[19] C. T. Kelley, Iterative methods for optimization, Society for Industrial and Applied Mathematics, Philadelphia, 1999.

[20] O. Kolb, Simulation and Optimization of Gas and Water Supply Networks, PhD thesis, TU Darmstadt, 2011.

[21] O. KOLB AND J. LANG, Mathematical optimization of water networks, vol. 162 of Internat. Ser. Numer. Math., Birkhäuser/Springer Basel AG, 2012, ch. Simulation and continuous optimization, pp. 17-33.

[22] J. Lebacque And M. Khoshyaran, First order macroscopic traffic flow models for networks in the context of dynamic assignment, in Transportation Planning, M. Patriksson and M. Labb, eds., vol. 64 of Applied Optimization, Springer US, 2002, pp. 119-140.

[23] M. J. Lighthill and G. B. Whitham, On Kinematic Waves. II. A Theory of Traffic Flow on Long Crowded Roads, Royal Society of London Proceedings Series A, 229 (1955), pp. 317-345.

[24] A. Muralidharan and R. Horowitz, Optimal control of freeway networks based on the Link Node Cell Transmission model, American Control Conference, (2012), pp. 5769-5774.

[25] M. Papageorgiou, H. Hadj-Salem, and J.-M. Blosseville, ALINEA: A local feedback control law for on-ramp metering, Transportation Research Record, 1320 (1991), pp. 58-64.

[26] J. Reilly, W. Krichene, M. L. D. Monache, S. Samaranayake, P. Goatin, AND A. M. BAYen, Adjoint-based optimization on a network of discretized scalar conservation law pdes with applications to coordinated ramp metering, submitted, (2014).

[27] P. Richards, Shock waves on the highway, Operations Research, 4 (1956), pp. $42-51$.

[28] P. Spellucci, Numerische Verfahren der Nichtlinearen Optimierung, Birkhäuser-Verlag, Basel, 1993. 
[29] — A new technique for inconsistent QP problems in the SQP method, Math. Methods Oper. Res., 47 (1998), pp. 355-400.

[30] — An SQP method for general nonlinear programs using only equality constrained subproblems, Mathematical Programming, 82 (1998), pp. 413448.

[31] M. Treiber and A. Kesting, Traffic flow dynamics, Springer, Heidelberg, 2013. Data, models and simulation, Translated by Treiber and Christian Thiemann. 